Check for updates

Cite this: RSC Adv., 2019, 9, 2948

Received 18th November 2018 Accepted 21st December 2018

DOI: $10.1039 / c 8 r a 09486 a$

rsc.li/rsc-advances

\section{Efficient full-colour organic light-emitting diodes based on donor-acceptor electroluminescent materials with a reduced singlet-triplet splitting energy gap $\dagger$}

\author{
Jayaraman Jayabharathi, (DD *a Ramaiyan Ramya, ${ }^{\text {* }}$ Venugopal Thanikachalam, (D) a \\ Palanivel Jeeva ${ }^{a}$ and Elayaperumal Sarojpurani ${ }^{a b}$
}

A series of efficient blue-emitting materials, namely, Cz-DPVI, Cz-DMPVI, Cz-DEPVI and TPA-DEPVI, possessing a donor-acceptor architecture with dual carrier transport properties and small singlet-triplet splitting is reported. These compounds exhibit excellent thermal properties with a very high glasstransition temperature $\left(T_{\mathrm{g}}\right)$, and thus, a stable uniform thin film was formed during device fabrication. Among the weak donor compounds, specifically, Cz-DPVI, Cz-DMPVI and Cz-DEPVI, the Cz-DEPVIbased device showed the maximum efficiencies $\left(L: 13955 \mathrm{~cd} \mathrm{~m}^{-2}, \eta_{\mathrm{ex}}: 4.90 \%, \eta_{\mathrm{c}}: 6.0 \mathrm{~cd} \mathrm{~A}^{-1}\right.$, and $\eta_{\mathrm{p}}: 5.4$ Im $\left.\mathrm{W}^{-1}\right)$ with CIE coordinates of $(0.15,0.06)$ at $2.8 \mathrm{~V}$. The electroluminescent efficiencies of Cz-DEPVI were higher than that of the strong donor TPA-DEPVI-based device $\left(L: 13856 \mathrm{~cd} \mathrm{~m}^{-2}, \eta_{\mathrm{ex}}: 4.70 \%, \eta_{\mathrm{c}}: 5.7\right.$

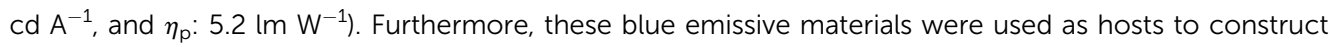
efficient green and red phosphorescent OLEDs. The green device based on Cz-DEPVI:Ir(ppy) 3 exhibited the maximum $L$ of $8891 \mathrm{~cd} \mathrm{~m}^{-2}, \eta_{\mathrm{ex}}$ of $19.3 \%, \eta_{\mathrm{c}}$ of $27.9 \mathrm{~cd} \mathrm{~A}^{-1}$ and $\eta_{\mathrm{p}}$ of $33.4 \mathrm{~lm} \mathrm{~W}^{-1}$ with CIE coordinates of $(0.31,0.60)$ and the red device based on Cz-DEPVI:Ir(MQ) $)_{2}$ (acac) exhibited the maximum $L$ of $40565 \mathrm{~cd} \mathrm{~m}^{-2}, \eta_{\mathrm{ex}}$ of $19.9 \%, \eta_{\mathrm{c}}$ of $26.0 \mathrm{~cd} \mathrm{~A}^{-1}$ and $\eta_{\mathrm{p}}$ of $27.0 \mathrm{~lm} \mathrm{~W}^{-1}$ with CIE coordinates of $(0.64,0.37)$.

\section{Introduction}

Currently, blue organic light-emitting devices (OLEDs) have attracted increasing interest due to their applications in FPD (flat panel displays) and SSL (solid state lighting). ${ }^{\mathbf{1 - 3}}$ However, the development of high-performance blue emissive materials with high fluorescent quantum yield $(\Phi)$, high thermal stability ( $T_{\mathrm{d}}$ and $T_{\mathrm{g}}$ ), good film-forming ability and balanced carrier injection/transporting properties remains challenging. ${ }^{4-6}$ Two important factors have been reported for $100 \%$ exciton utilization efficiency $\left(\eta_{\mathrm{S}}\right)$ : (i) thermally activated delayed fluorescence (TADF) and (ii) hybrid local and charge transfer (HLCT) state (Scheme 1). ${ }^{7}$ Although high external quantum efficiencies have been obtained from blue $(37.5 \%)$, green $(31.3 \%)$ and red (17.5\%) TADF OLEDs, their fabrication is expensive since the long lifetime of the $\mathrm{T} 1$ state in the TADF process suffers from exciton annihilation. ${ }^{\mathbf{8 9}}$ Organic donor-acceptor (D-A) compounds with hybridized local and charge transfer exhibit high $\eta_{\mathrm{S}}$ in fluorescent OLEDs, which can be attributed to the hot

${ }^{a}$ Department of Chemistry, Annamalai University, Annamalai Nagar, Tamilnadu, 608 002, India

${ }^{b}$ Sri Manakula Vinayagar Engineering College, India

$\dagger$ Electronic supplementary information (ESI) available. See DOI: 10.1039/c8ra09486a exciton mechanism. ${ }^{\mathbf{1 0 - 1 6}}$ When the LE and CT states are close in energy, mixing of the LE and CT states is possible as a linear combination of both states $\left(\psi(\mathrm{CT})\right.$ and $\psi(\mathrm{LE})$, i.e., $\Psi\left(\mathrm{S}_{1}\right)=\Psi(\mathrm{LE})$ $+\lambda \times \Psi(\mathrm{CT}))$ and $\lambda=\left|\left\langle\Psi_{\mathrm{LE}}|H| \Psi_{\mathrm{CT}}\right\rangle / E_{\mathrm{CT}}-E_{\mathrm{LE}}\right|$. The low-lying LE-dominated HLCT state (high \% LE) provides a high radiative rate $\left(k_{\mathrm{r}}\right)$ for high photoluminescence efficiency $\left(\eta_{\mathrm{PL}}\right)$; whereas, the high-lying CT (high \% CT) dominated HLCT state is responsible for high $\eta_{\mathrm{S}}$ through the RISC process together with the hot exciton mechanism. ${ }^{\mathbf{1 4 - 1 7}}$

The external quantum efficiency $\left(\eta_{\mathrm{EQE}}\right)$ and exciton utilization efficiency $\left(\eta_{\mathrm{S}}\right)$ can be calculated as follows: $\eta_{\mathrm{EQE}}=\eta_{\text {out }} \times \eta_{\mathrm{IQE}}$ $=\eta_{\text {out }} \times \eta_{\text {rec }} \times \eta_{\gamma} \times \Phi_{\mathrm{PL}}$ and $\eta_{\mathrm{S}}=\eta_{\text {out }} \times \eta_{\text {rec }} \times \eta_{\mathrm{PL}}$, where, $\eta_{\mathrm{IQE}}$ is the internal quantum efficiency, $\eta_{\text {out }}$ the light outcoupling efficiency $\left(1 / 2 n^{2}, n=1.5, \eta_{\text {out }} \sim 20 \%\right), \eta_{\text {rec }}$ the efficiency for electron hole recombination $(100 \%), \eta_{\mathrm{PL}}$ the photoluminescence efficiency of the film and $\eta_{\mathrm{S}}$ the exciton utilization efficiency. ${ }^{18}$ Presently, the construction of emissive states consisting of both LE and CT components for high PL efficiency ( $\eta_{\mathrm{PL}}$ : LE state) and high exciton utilization ( $\eta_{\mathrm{S}}$ : from CT state) is interest. ${ }^{19}$ According to the energy gap law, a larger energy gap between the $T_{2}$ and $T_{1}$ states reduces the internal conversion (IC) $\left(\mathrm{T}_{2} \stackrel{k_{\mathrm{IC}}}{\rightarrow} \mathrm{T}_{1}\right)$, which results in hot RISC $\left(\mathrm{T}_{1} \stackrel{k_{\mathrm{RISC}}}{\longrightarrow} \mathrm{S}_{1} / \mathrm{S}_{2}\right)$ rather than cold RISC $\left(\mathrm{T}_{1} \stackrel{k_{\text {RISC }}}{\longrightarrow} \mathrm{S}_{1}\right){ }^{20}$ Therefore, the hot exciton mechanism with the HLCT state increases the $\eta_{\mathrm{EQE}}$ as a result of the coexistence of high $\eta_{\mathrm{PL}}$ and high $\eta_{\mathrm{s}}$. A blue emissive material with balanced 


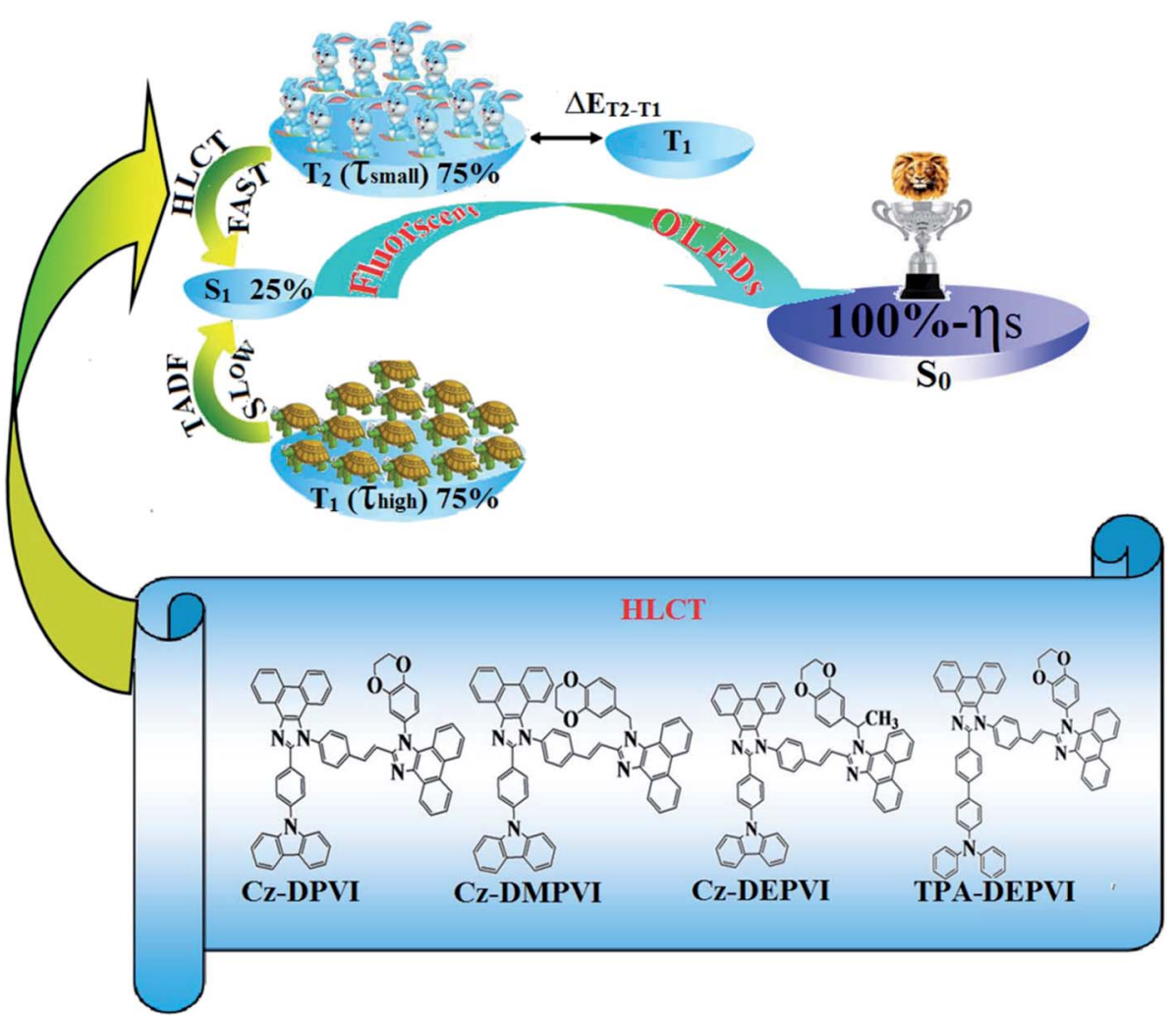

Scheme 1 Effect of TADF and HLCT on 100\% exciton utilization efficiency $\left(\eta_{\mathrm{S}}\right)$.

carrier transport characteristics and high triplet energy $\left(E_{\mathrm{T}}\right)$ may be employed as a host for green and red phosphorescent emitters. However, high-performance non-doped blue electroluminescent materials are not a suitable host for phosphorescent OLEDs due to their low $E_{\mathrm{T}}$ and poor carrier transport properties. ${ }^{3}$ An efficient host for green and red phosphors exhibits low efficiency when used as blue OLEDs. ${ }^{21}$ Therefore, it is still challenging to achieve efficient full-color OLEDs with blue emissive materials. Herein, we report the use of multi-functional organic OLED materials (Cz-DPVI, Cz-DMPVI, Cz-DEPVI and TPA-DEPVI) as (i) emitters in blue OLEDs and (ii) hosts for green and red OLEDs. These materials consist of a hole transport moiety (donor) and an electron transport moiety (acceptor) with high quantum yield in film $\left(\phi_{\text {film }}\right)$ with small singlet-triplet splitting $\left(\Delta_{\mathrm{ST}}\right)$ to ensure that the triplet excited state energy $\left(E_{\mathrm{T}}\right)$ is high enough to excite the green and red phosphorescent dopant. The $\mathrm{H}-\mathrm{H}$ repulsion of the styryl fragment (i) with a phenanthrene part and dihydrobenzodioxin and with a 9-( $p$-tolyl)-9H-carbazole moiety (Cz-DPVI, Cz-DMPVI and Cz-DEPVI)/phenyl of the TPA moiety (TPA-DEPVI) leads to a twisted configuration, which enhanced the twist angle, and thus, shortened the conjugation length. The solvatochromic effect of Cz-DPVI, Cz-DMPVI, CzDEPVI and TPA-DEPVI was studied to understand the excited state characteristics and interstate coupling strength of the LE $\left[\left(\mathrm{D}^{-} \mathrm{A}^{*}\right) /\left(\mathrm{D}^{*}-\mathrm{A}\right)\right]$ and $\mathrm{CT}\left[\left(\mathrm{D}^{+}-\mathrm{A}^{-}\right)\right]$components. The LE and CT composition in the single emissive state was discussed using natural transition orbital (NTO), centroids of charges and transition density matrix (TDM) analysis. The hybridization of the LE and CT energy states was used for the molecular design and their composition in HLCT was tuned, which resulted in high EL efficiency. The weak electron-donating carbazole (Cz) in Cz-DPVI, Cz-DMPVI and Cz-DEPVI decreased the \% CT with an increase in the \% LE composition in the $\mathrm{S}_{1}$ state; whereas, in TPA-DEPVI, the extended $\pi$-conjugation (increased $\%$ LE) resulted in an enhancement in PL efficiency $\left(\eta_{\mathrm{PL}}\right)$ while simultaneously maintaining donor strength (\% LE). Among the weak donor compounds, specifically, Cz-DPVI, Cz-DMPVI and Cz-DEPVI, the Cz-DEPVI-based device showed the maximum efficiencies $(L$ : $13955 \mathrm{~cd} \mathrm{~m}^{-2}, \eta_{\mathrm{ex}}: 4.90 \%, \eta_{\mathrm{c}}: 6.0 \mathrm{~cd} \mathrm{~A}^{-1}$, and $\eta_{\mathrm{p}}: 5.4 \mathrm{~lm} \mathrm{~W}^{-1}$ ) with CIE coordinates of $(0.15,0.06)$ at $2.0 \mathrm{~V}$. The electroluminescence efficiencies of Cz-DEPVI were higher than that of the strong donor TPA-DEPVI-based device ( $L: 13856 \mathrm{~cd} \mathrm{~m}^{-2}, \eta_{\mathrm{ex}}: 4.70 \%, \eta_{\mathrm{c}}$ : $5.7 \mathrm{~cd} \mathrm{~A}^{-1}$, and $\eta_{\mathrm{p}}: 5.2 \mathrm{~lm} \mathrm{~W}^{-1}$ ). The green device based on $\mathrm{Cz}^{-}$ DEPVI:Ir(ppy $)_{3}$ exhibited the maximum $L$ of $8891 \mathrm{~cd} \mathrm{~m}^{-2}, \eta_{\text {ex }}$ of $19.3 \%, \eta_{\mathrm{c}}$ of $27.9 \mathrm{~cd} \mathrm{~A}^{-1}$ and $\eta_{\mathrm{p}}$ of $33.4 \mathrm{~lm} \mathrm{~W} \mathrm{~m}^{-1}$ with CIE coordinates of $(0.31,0.60)$ and the red device based on $\mathrm{Cz}^{-}$ DEPVI:Ir(MQ) $)_{2}(\mathrm{acac})$ exhibited the maximum $L$ of $40565 \mathrm{~cd} \mathrm{~m}^{-2}$, $\eta_{\mathrm{ex}}$ of $19.9 \%$, of $\eta_{\mathrm{c}}$ of $26.0 \mathrm{~cd} \mathrm{~A}^{-1}$ and $\eta_{\mathrm{p}}$ of $27.0 \mathrm{~lm} \mathrm{~W}^{-1}$ with CIE coordinates of $(0.64,0.37)$. These results are highly useful for the design of low-cost fluorescent materials using subtle molecular modification and the HLCT emissive state principle.

\section{Experimental}

\subsection{Measurements and general methods}

The reagents and solvents used for synthesis (Scheme 2) were purchased from Sigma-Aldrich. ${ }^{1} \mathrm{H}$ and ${ }^{13} \mathrm{C}$ NMR spectra were recorded with a Bruker $400 \mathrm{MHz}$ spectrometer and mass spectra 


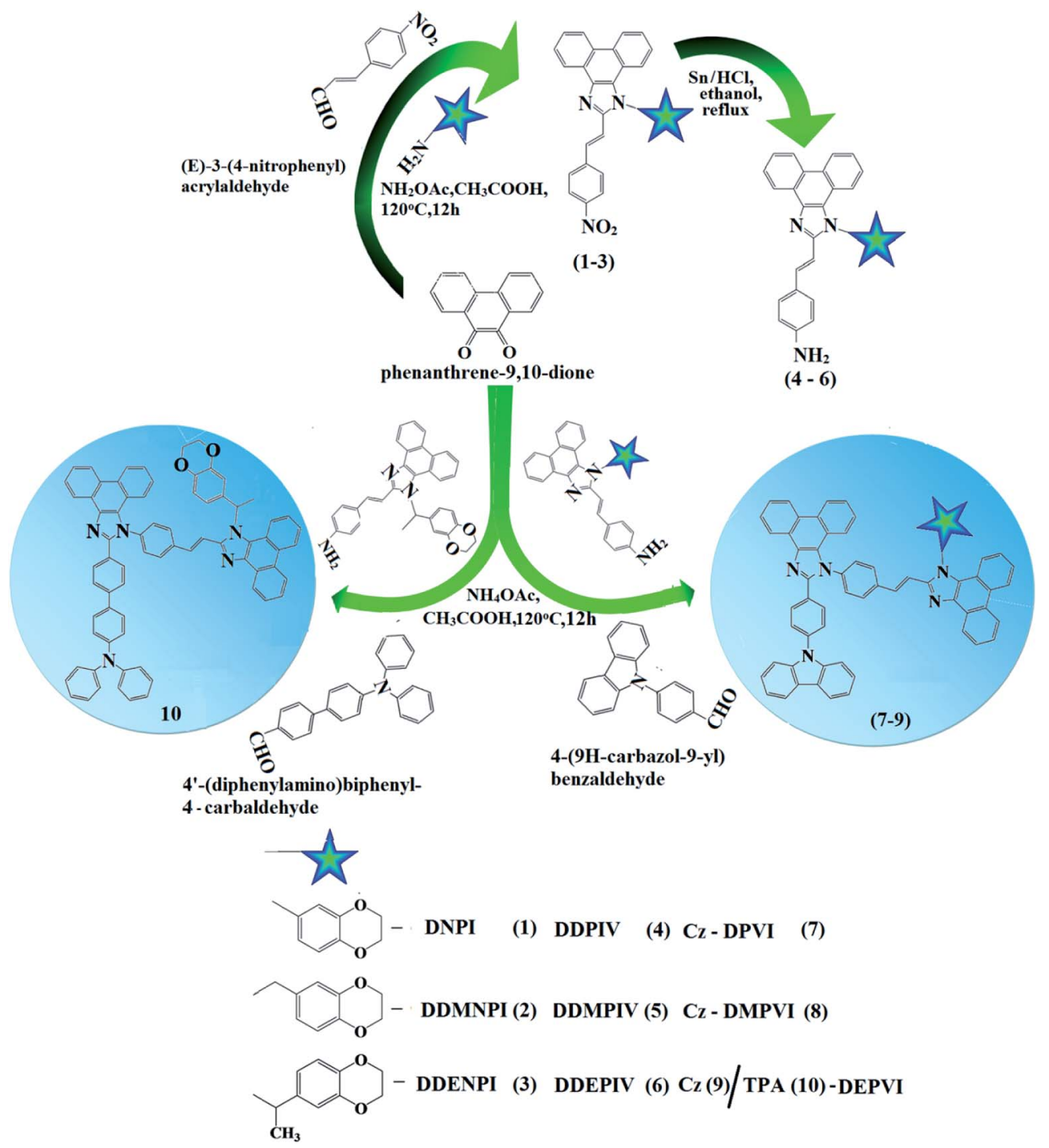

Scheme 2 Synthetic route for Cz-DPVI, Cz-DMPVI, Cz-DEPVI and TPA-DEPVI.

with an Agilent spectrometer (LCMS VL SD). UV-vis absorption spectra in solution and film were measured on a Perkin-Elmer Lambda 35 and Lambda 35 spectrophotometer with an integrated sphere (RSA-PE-20), respectively. Photoluminescence (PL) studies were carried out using a PerkinElmer LS55 fluorescence spectrometer. Thermogravimetric analysis (TGA) and differential scanning calorimetry (DSC) were performed on a PerkinElmer thermal analysis system and NETZSCH-DSC-204, respectively, with a heating rate of $10{ }^{\circ} \mathrm{C} \mathrm{min}{ }^{-1}$ and $\mathrm{N}_{2}$ flow rate of $100 \mathrm{~mL} \mathrm{~min}^{-1}$ for both. The fluorescence lifetimes of the emissive materials were estimated from the time-resolved fluorescence decay spectra obtained via the time-correlated single-photon counting (TCSPC) method on a Horiba Fluorocube-01-NL lifetime system equipped with a Nano LED excitation source and TBX-PS detector. The DAS6 software was used to analyse decay via the reconvolution method and goodness of fit was determined using reduced $\chi^{2}$ values. The absolute quantum yield (PLQY) was determined using an F7100 fluorescence spectrometer. Cyclic voltammetry was performed using a potentiostat CHI 630A electrochemical analyzer (platinum electrode and platinum wire as the working electrode and counter electrode, respectively, $\mathrm{Ag} / \mathrm{Ag}^{+}$as the reference electrode, and scan rate of $100 \mathrm{mV} \mathrm{s}^{-1}$ ). Ferrocene was used as the internal standard with the highest occupied molecular orbital energy (HOMO) of $-4.80 \mathrm{eV}$ and $0.1 \mathrm{M}$ tetrabutylammoniumperchlorate in $\mathrm{CH}_{2} \mathrm{Cl}_{2}$ as the supporting electrolyte. The HOMO energies were calculated by measuring the oxidation potentials $\left[E_{\text {HOMO }}=-\left(E_{\mathrm{ox}}+4.8 \mathrm{eV}\right)\right]$ and the LUMO energies were estimated by subtracting the optical band gap from the HOMO energies $\left[E_{\text {LUMO }}=E_{\text {HOMO }}-1239 / \lambda_{\text {onset }}\right]$.

\subsection{Computational details}

For the theoretical calculations, the ground state (DFT)/excited state (TD-DFT) geometrical properties were optimized by employing the Gaussian 09 program. ${ }^{22}$ The multifunctional wavefunction analyzer (Multiwfn) ${ }^{22}$ was used to determine the 
nature of the electronic transitions of the excited states and natural transition orbitals (NTOs).

\subsection{Synthesis of $(E)-1-(2,3-d i h y d r o b e n z o[b][1,4]$ dioxin-6-yl)- 2-(4-nitrostyryl)-1H-phenanthro[9,10- $d]$ imidazole (DDNPI)}

A mixture of phenanthrenequinone (2.08 g, $10 \mathrm{mmol})$, 4-nitrocinnamaldehyde (1.51 g, $10 \mathrm{mmol})$, 1,4-benzodioxane-6-amine (4.65 g, $50 \mathrm{mmol}$ ) and ammonium acetate $(3.08 \mathrm{~g}, 40 \mathrm{mmol})$ in acetic acid $(25 \mathrm{~mL})$ was refluxed $\left(120^{\circ} \mathrm{C}, 12 \mathrm{~h}\right.$, and $\mathrm{N}_{2}$ stream). The reaction mixture was cooled and poured into methanol. The separated crude product was purified by column chromatography using hexane:ethylacetate as the eluent (Scheme 2). Yield: $60 \%$. Anal. calcd for $\mathrm{C}_{31} \mathrm{H}_{21} \mathrm{~N}_{3} \mathrm{O}_{4}: \mathrm{C}, 74.53 ; \mathrm{H}, 4.25 ; \mathrm{N}$, 8.41. Found: C, 74.50; H, 4.21; N, 8.35. ${ }^{1} \mathrm{H}$ NMR $(400 \mathrm{MHz}$, $\left.\mathrm{CDCl}_{3}\right): \delta 4.18-4.36(\mathrm{~m}, 4 \mathrm{H}), 6.80(\mathrm{~d}, J=16.2 \mathrm{~Hz}, 1 \mathrm{H}), 6.88(\mathrm{t}$, $1 \mathrm{H}), 7.12(\mathrm{~d}, J=15.0 \mathrm{~Hz}, 1 \mathrm{H}), 7.28$ (d, $J=8.0 \mathrm{~Hz}, 1 \mathrm{H}), 7.25-7.39$ (m, 5H), 7.60-7.69 (m, 5H), $8.05(\mathrm{~s}, 1 \mathrm{H}), 8.59(\mathrm{t}, 1 \mathrm{H}), 8.75(\mathrm{~s}, 1 \mathrm{H})$. ${ }^{13} \mathrm{C}$ NMR (100 MHz, $\mathrm{CDCl}_{3}$ ): $\delta$ 64.30, 64.32, 102.53, 110.82, 114.76, 121.12, 122.42, 126.53, 127.31, 127.69, 127.94, 128.39, 131.43, 131.54, 133.39, 141.25, 141.57, 146.09, 147.61, 147.61. MS: $m / z .499 .08\left[\mathrm{M}^{+}\right]$; calcd 499.26.

\subsection{Synthesis of 4-((E)-2-(1-(2,3-dihydrobenzo $[b][1,4]$ dioxin- 6-yl)-1H-phenanthro[9,10- $d]$ imidazol-2-yl)vinyl)benzenamine (DDPIV)}

A mixture of (E)-1-(2,3-dihydrobenzo[b][1,4]dioxin-6-yl)-2-(4nitrostyryl)-1 $H$-phenanthro[9,10- $d]$ imidazole (DDNPI) (4.15 g, $10 \mathrm{mmol}$ ) and $10 \% \mathrm{Sn} / \mathrm{HCl}(250 \mathrm{mg})$ in $25 \mathrm{~mL}$ ethanol was refluxed under stirring and $80 \%$ hydrazine hydrate $(15 \mathrm{~mL})$ was added dropwise within $30 \mathrm{~min}$ and stirring was continued for $14 \mathrm{~h}$. The reaction mixture was neutralized with aq. $\mathrm{HCl}$ and the obtained white product was recrystallized from an ethanol : water mixture. Yield: $58 \%$. Anal. calcd for $\mathrm{C}_{31} \mathrm{H}_{23} \mathrm{~N}_{3} \mathrm{O}_{2}$ : C, 79.31; H, 4.92; N, 8.94. Found: C, 79.28; H, 4.88; N, 8.89. ${ }^{1} \mathrm{H}$ NMR $(400$ $\left.\mathrm{MHz}, \mathrm{CDCl}_{3}\right) \delta 4.01(\mathrm{~s}, 2 \mathrm{H}), 4.10-4.30(\mathrm{~m}, 4 \mathrm{H}), 6.71(\mathrm{~d}, J=$ $16.0 \mathrm{~Hz}, 1 \mathrm{H}), 6.52(\mathrm{~s}, 2 \mathrm{H}), 6.95(\mathrm{~d}, J=8.2 \mathrm{~Hz}, 1 \mathrm{H}), 6.98(\mathrm{~d}, J=$ $16.2 \mathrm{~Hz}, 1 \mathrm{H}), 6.85-6.93(\mathrm{~m}, 6 \mathrm{H}), 7.05(\mathrm{~s}, 2 \mathrm{H}), 7.82(\mathrm{~d}, J=8.2 \mathrm{~Hz}$, $1 \mathrm{H}), 8.13(\mathrm{~d}, J=8.4 \mathrm{~Hz}, 2 \mathrm{H}), 8.90(\mathrm{~d}, J=7.6 \mathrm{~Hz}, 2 \mathrm{H}) .{ }^{13} \mathrm{C} \mathrm{NMR}$ $\left(100 \mathrm{MHz}, \mathrm{CDCl}_{3}\right): \delta 63.35,101.54,112.82,115.93,116.20$, $122.43,125.21,126.01,126.22,126.56,126.82,127.63,128.35$, 131.41, 133.43, 141.49, 146.21, 147.60, 147.21. MS: $\mathrm{m} / \mathrm{z} .469 .10$ $\left[\mathrm{M}^{+}\right]$; calcd 469.26.

2.5. Synthesis of 2-(4-(9H-carbazol-9-yl)phenyl)-1-(4-((E)-2-(1(2,3-dihydrobenzo $[b][1,4]$ dioxin-8-yl)-1H-phenanthro[9,10- $d]$ imidazol-2-yl)vinyl)phenyl)-1H-phenanthro[9,10- $d]$ imidazole (Cz-DPVI)

A mixture of phenanthrenequinone $(0.416 \mathrm{~g}, 2 \mathrm{mmol}), 4-\left(9 \mathrm{H}^{-}\right.$ carbazol-9-yl)benzaldehyde (0.698 g, $2 \mathrm{mmol}), 4-((E)-2-(1-(2,3-$ dihydrobenzo[ $b]$ rdioxin-6-yl)-1 $H$-phenanthro[9,10- $d]$ imidazol-2yl)vinyl)benzenamine (DDPIV) (1.16 g, $3 \mathrm{mmol}$ ) and ammonium acetate $(1.54 \mathrm{~g}, 20 \mathrm{mmol})$ in glacial acetic acid $(25 \mathrm{~mL})$ was refluxed $\left(140{ }^{\circ} \mathrm{C}, 20 \mathrm{~h}\right.$, and $\mathrm{N}_{2}$ stream $) .{ }^{23-25}$ The reaction mixture was poured into methanol and the separated white solid was filtered, washed with water and purified by column chromatography using $\mathrm{CH}_{2} \mathrm{Cl}_{2}$ as the eluent. Yield: $60 \%$. Anal. calcd for
$\mathrm{C}_{64} \mathrm{H}_{41} \mathrm{~N}_{5} \mathrm{O}_{2}$ : C, 84.28; H, 4.52; N, 7.65. Found: C, 84.19; $\mathrm{H}$, 4.48; N, 7.31. ${ }^{1} \mathrm{H}$ NMR (400 $\left.\mathrm{MHz} \mathrm{CDCl}_{3}\right) \delta 4.18-4.32(\mathrm{~m}, 4 \mathrm{H})$, $6.73(\mathrm{~d}, J=15.2 \mathrm{~Hz}, 2 \mathrm{H}), 6.70(\mathrm{~s}, 1 \mathrm{H}), 6.99(\mathrm{~s}, 2 \mathrm{H}), 7.01-7.50(\mathrm{~m}$, $8 \mathrm{H}), 7.05-7.41(\mathrm{~m}, 8 \mathrm{H}), 7.62-7.72(\mathrm{~m}, 10 \mathrm{H}), 7.54(\mathrm{~d}, J=16.2 \mathrm{~Hz}$, $2 \mathrm{H}), 8.33$ (d, $J=7.8 \mathrm{~Hz}, 2 \mathrm{H}), 8.65(\mathrm{t}, 2 \mathrm{H}) .{ }^{13} \mathrm{C}$ NMR $(100 \mathrm{MHz}$, $\left.\mathrm{CDCl}_{3}\right): \delta 64.21,101.32,111.10,119.21,120.01,122.21,122.35$, 122.42, 126.01, 126.21, 126.43, 126.60, 127.31, 128.13, 128.30, 129.60, 131.54, 133.41, 139.71, 141.31, 146.21, 147.65. MS: $m / z$. $910.29\left[\mathrm{M}^{+}\right]$; calcd 910.35 .

\subsection{Synthesis of $(E)-1-((2,3-d i h y d r o b e n z o[b][1,4]$ dioxin-6-yl)} methyl)-2-(4-nitrostyryl)-1H-phenanthro[9,10- $d]$ imidazole (DDMNPI)

DDMNPI was synthesized using (2,3-dihydrobenzo[b][1,4] dioxin-6-yl)methanamine following a procedure similar to that of DDNPI. Yield: $62 \%$. Anal. calcd for $\mathrm{C}_{32} \mathrm{H}_{23} \mathrm{~N}_{3} \mathrm{O}_{4}$ : C, 74.82; $\mathrm{H}$, 4.50; N, 8.17. Found: C, 74.78; H, 4.41; N, 8.10. ${ }^{1} \mathrm{H}$ NMR (400 $\left.\mathrm{MHz}, \mathrm{CDCl}_{3}\right): \delta 4.36(\mathrm{~m}, 4 \mathrm{H}), 4.99(\mathrm{~s}, 2 \mathrm{H}) 6.46(\mathrm{~d}, J=16.4 \mathrm{~Hz}$, $1 \mathrm{H}), 6.51(\mathrm{~d}, J=8.0 \mathrm{~Hz}, 1 \mathrm{H}), 7.13(\mathrm{~d}, J=15.0 \mathrm{~Hz}, 1 \mathrm{H}), 7.25(\mathrm{~d}, J=$ $8.0 \mathrm{~Hz}, 1 \mathrm{H}), 7.24-7.40(\mathrm{~m}, 5 \mathrm{H}), 7.62-7.65(\mathrm{~m}, 5 \mathrm{H}), 8.15(\mathrm{~s}, 1 \mathrm{H})$, $8.68(\mathrm{t}, 1 \mathrm{H}), 8.79(\mathrm{~s}, 1 \mathrm{H}) .{ }^{13} \mathrm{C} \mathrm{NMR}\left(100 \mathrm{MHz}, \mathrm{CDCl}_{3}\right): \delta 50.2$, $64.32,102.58,110.85,114.67,121.19,122.48,126.57,126.32$, $127.30,127.65,131.49,131.54,133.37,141.29,141.67,146.18$, 147.63, 147.68. MS: m/z. $513.10\left[\mathrm{M}^{+}\right]$; calcd 513.19.

2.7. Synthesis of $(E)-4-(2-(1-((2,3-d i h y d r o b e n z o[b][1,4]$ dioxin6-yl)methyl)-1H-phenanthro[9,10-d]imidazol-2-yl)vinyl)aniline (DDMPIV)

DDMPIV was synthesized using DDMNPI following a procedure similar to that of DDMNPI. Yield: $60 \%$. Anal. calcd for $\mathrm{C}_{32} \mathrm{H}_{25} \mathrm{~N}_{3} \mathrm{O}_{2}$ : C, 79.48; H, 5.22; N, 8.69. Found: C, 79.32; H, 5.18; N, 8.58. ${ }^{1} \mathrm{H}$ NMR (400 MHz, $\left.\mathrm{CDCl}_{3}\right) \delta 4.05(\mathrm{~s}, 2 \mathrm{H}), 4.39(\mathrm{~m}$, $4 \mathrm{H}), 4.99(\mathrm{~s}, 2 \mathrm{H}), 6.46(\mathrm{~d}, J=16.0 \mathrm{~Hz}, 1 \mathrm{H}), 6.53(\mathrm{~s}, 2 \mathrm{H}), 6.89(\mathrm{~d}, J$ $=8.0 \mathrm{~Hz}, 1 \mathrm{H}), 6.99(\mathrm{~d}, J=16.2 \mathrm{~Hz}, 1 \mathrm{H}), 6.75-6.98(\mathrm{~m}, 6 \mathrm{H}), 7.05$ (s, 2H), $7.84(\mathrm{~d}, J=16.0 \mathrm{~Hz}, 1 \mathrm{H}), 8.14(\mathrm{~d}, J=8.0 \mathrm{~Hz}, 2 \mathrm{H}), 8.95$ (d, $J=7.0 \mathrm{~Hz}, 2 \mathrm{H}) .{ }^{13} \mathrm{C} \mathrm{NMR}\left(100 \mathrm{MHz}, \mathrm{CDCl}_{3}\right): \delta$ 50.25, 63.38, $112.85,114.93,116.25,121.38,126.31,126.43,126.52,126.56$, $126.72,127.73,127.85,128.41,131.43,133.49,141.23,143.60$, 146.21, 147.28 MS: $\mathrm{m} / z .483 .53\left[\mathrm{M}^{+}\right]$; calcd 483.62.

2.8. Synthesis of 2-(4-(9H-carbazol-9-yl)phenyl)-1-(4-((E)-2-(1((2,3-dihydrobenzo[b][1,4]dioxin-6-yl)methyl)-1 $H$-phenanthro $[9,10-d]$ imidazol-2-yl)vinyl)phenyl)-1H-phenanthro[9,10- $d]$ imidazole (Cz-DMPVI)

Cz-DMPVI was synthesized using DDMNPI following a procedure similar to that of Cz-DPVI. Yield: 58\%. Anal. calcd for $\mathrm{C}_{65} \mathrm{H}_{43} \mathrm{~N}_{5} \mathrm{O}_{2}$ : C, 84.28; H, 4.52; N, 7.65. Found: C, 84.19; H, 4.48; N, 7.31. ${ }^{1} \mathrm{H}$ NMR (400 MHz, $\left.\mathrm{CDCl}_{3}\right) \delta 4.32(\mathrm{~m}, 4 \mathrm{H}), 6.46(\mathrm{~s}$, $1 \mathrm{H}), 6.99(\mathrm{~d}, 1 \mathrm{H}), 6.75(\mathrm{~d}, J=16.0 \mathrm{~Hz}, 2 \mathrm{H}), 6.68(\mathrm{~s}, 1 \mathrm{H}), 6.95(\mathrm{~s}$, 2H), 7.02-7.59 (m, 9H), 7.05-7.48 (m, 7H), 7.60-7.79 (m, 10H), $7.56(\mathrm{~d}, J=8.8 \mathrm{~Hz}, 2 \mathrm{H}), 8.38(\mathrm{~d}, J=8.0 \mathrm{~Hz}, 2 \mathrm{H}), 8.67(\mathrm{t}, 2 \mathrm{H}) .{ }^{13} \mathrm{C}$ NMR (100 MHz, $\mathrm{CDCl}_{3}$ ): $\delta$ 50.29, 63.98, 111.12, 111.19, 120.01, 122.25, 122.38, 122.46, 126.01, 126.22, 126.44, 126.62, 127.32, 128.15, 128.38, 129.65, 131.59, 133.39, 139.89, 141.38, 146.27, 147.68. MS: $m / z .925 .27\left[\mathrm{M}^{+}\right]$; calcd 925.38 . 
2.9. Synthesis of $(E)-1-(1-(2,3-d i h y d r o b e n z o[b][1,4]$ dioxin-6yl)ethyl)-2-(4-nitrostyryl)-1H-phenanthro[9,10- $d]$ imidazole (DDENPI)

DDENPI was synthesized using 1-(2,3-dihydrobenzo[b][1,4] dioxin-6-yl)ethanamine following a procedure similar to that of DDNPI. Yield: $62 \%$. Anal. calcd for $\mathrm{C}_{33} \mathrm{H}_{25} \mathrm{~N}_{3} \mathrm{O}_{4}$ : C, 75.12; $\mathrm{H}$, 4.75; N, 7.93. Found: C, 75.01; H, 4.68; N, 7.87. ${ }^{1} \mathrm{H}$ NMR $(400$ $\left.\mathrm{MHz}, \mathrm{CDCl}_{3}\right): \delta 1.90(\mathrm{~s}, 3 \mathrm{H}), 4.38(\mathrm{~m}, 4 \mathrm{H}), 5.16(\mathrm{~d}, J=8.8 \mathrm{~Hz}$, $1 \mathrm{H}), 6.53(\mathrm{~d}, J=15.0 \mathrm{~Hz}, 1 \mathrm{H}), 6.51(\mathrm{~d}, J=8.0 \mathrm{~Hz}, 1 \mathrm{H}), 7.14(\mathrm{~d}, J=$ $7.0 \mathrm{~Hz}, 1 \mathrm{H}), 7.28(\mathrm{~d}, J=8.0 \mathrm{~Hz}, 1 \mathrm{H}), 7.34-7.56(\mathrm{~m}, 6 \mathrm{H}), 7.68-7.68$ $(\mathrm{m}, 4 \mathrm{H}), 8.12(\mathrm{~s}, 1 \mathrm{H}), 8.14(\mathrm{t}, 1 \mathrm{H}), 8.89(\mathrm{~s}, 1 \mathrm{H}) .{ }^{13} \mathrm{C}$ NMR $(100$ $\left.\mathrm{MHz}, \mathrm{CDCl}_{3}\right): \delta 22.31,54.01,64.33,112.53,112.82,114.98$, 121.02, 121.09, 122.43, 126.61, 126.68, 126.73, 131.53, 133.49, 136.68, 141.33, 141.58, 143.93, 146.63, 147.65. MS: $\mathrm{m} / \mathrm{z} .527 .12$ $\left[\mathrm{M}^{+}\right]$; calcd 527.01.

\subsection{Synthesis of $(E)-4-(2-(1-(1-(2,3-$ dihydrobenzo $[b][1,4]$ dioxin-6-yl)ethyl)-1H-phenanthro[9,10-d]imidazol-2-yl)vinyl) aniline (DDEPIV)}

DDEPIV was synthesized using DDENPI following a procedure similar to that of DDMNPI. Yield: 50\%. Anal. calcd for $\mathrm{C}_{33} \mathrm{H}_{27} \mathrm{~N}_{3} \mathrm{O}_{2}$ : C, 79.63; H, 5.15; N, 6.57. Found: C, 79.58; $\mathrm{H}$, 5.08; N, 6.48. ${ }^{1} \mathrm{H}$ NMR (400 MHz, $\left.\mathrm{CDCl}_{3}\right): \delta 1.83(\mathrm{~s}, 3 \mathrm{H}), 4.03(\mathrm{~s}$, $2 \mathrm{H}), 4.37(\mathrm{~m}, 4 \mathrm{H}), 5.17(\mathrm{~d}, J=8.0 \mathrm{~Hz}, 1 \mathrm{H}), 6.50(\mathrm{~d}, J=16.0 \mathrm{~Hz}$, $1 \mathrm{H}), 6.54(\mathrm{~d}, J=8.6 \mathrm{~Hz}, 1 \mathrm{H}), 7.18(\mathrm{~d}, J=8.0 \mathrm{~Hz}, 1 \mathrm{H}), 7.29(\mathrm{~d}, J=$ $16.0 \mathrm{~Hz}, 1 \mathrm{H}), 7.34-7.56(\mathrm{~m}, 4 \mathrm{H}), 7.65-7.77(\mathrm{~m}, 6 \mathrm{H}), 8.10(\mathrm{~s}, 1 \mathrm{H})$, $8.18(\mathrm{t}, 1 \mathrm{H}), 8.80(\mathrm{~s}, 1 \mathrm{H}) .{ }^{13} \mathrm{C}$ NMR $\left(100 \mathrm{MHz}, \mathrm{CDCl}_{3}\right): \delta 21.99$, $53.01,63.98,112.58,112.86,114.97,121.05,121.10,122.45$, 126.60, 126.65, 126.78, 131.54, 133.47, 136.65, 141.34, 141.56, 143.90, 146.67, 147.69. MS: m/z. $497.18\left[\mathrm{M}^{+}\right]$; calcd 497.27.

2.11. Synthesis of 2-(4-(9H-carbazol-9-yl)phenyl)-1-(4-((E)-2(1-(1-(2,3-dihydrobenzo[b][1,4]dioxin-7-yl)ethyl)-1Hphenanthro[9,10- $d]$ imidazol-2-yl)vinyl)phenyl)-1Hphenanthro[9,10-d] imidazole (Cz-DEPVI)

Cz-DEPVI was synthesized using DDMNPI following a procedure similar to that of Cz-DPVI yield: $50 \%$. Anal. calcd for $\mathrm{C}_{66} \mathrm{H}_{45} \mathrm{~N}_{5} \mathrm{O}_{2}$ : C, 84.32; H, 4.80; N, 7.42. Found: C, 84.25; $\mathrm{H}$, 4.75; N, 7.35. ${ }^{1} \mathrm{H}$ NMR (400 MHz, $\left.\mathrm{CDCl}_{3}\right) \delta 1.88(\mathrm{~s}, 3 \mathrm{H}), 4.33(\mathrm{~m}$, $4 \mathrm{H}), 5.15(\mathrm{~d}, J=8.0 \mathrm{~Hz}, 1 \mathrm{H}), 6.57(\mathrm{~s}, 1 \mathrm{H}), 6.95(\mathrm{~d}, 1 \mathrm{H}), 6.78(\mathrm{~d}, J=$ 15.0 Hz, 2H), 6.65 (s, 1H), 6.93 (s, 2H), 7.01-7.55 (m, 10H), 7.05$7.45(\mathrm{~m}, 7 \mathrm{H}), 7.63-7.81(\mathrm{~m}, 14 \mathrm{H}), 7.58(\mathrm{~d}, J=8.0 \mathrm{~Hz}, 2 \mathrm{H}), 8.39(\mathrm{~d}$, $J=8.8 \mathrm{~Hz}, 2 \mathrm{H}), 8.63(\mathrm{t}, 2 \mathrm{H}) .{ }^{13} \mathrm{C} \mathrm{NMR}\left(100 \mathrm{MHz}, \mathrm{CDCl}_{3}\right): \delta 21.98$, 53.29 , 64.01, 111.13, 111.18, 120.02, 122.29, 122.35, 122.45, 126.09, 126.28, 126.44, 126.66, 127.33, 128.11, 128.37, 129.66, 131.55, 133.35, 139.88, 141.37, 146.22, 147.63. MS: $\mathrm{m} / \mathrm{z} .938 .38$ $\left[\mathrm{M}^{+}\right]$; calcd 938.45.

2.12. (E)-4'-(1-(4-(2-(1-((2,3-Dihydrobenzo $[b][1,4]$ dioxin-6-yl) ethyl)-1 $H$-phenanthro[9,10-imidazol-2-yl)vinyl)phenyl)-1 $H$ phenanthro[9,10- $d]$ imidazol-2-yl)- $N, N$-diphenyl- $\left[1,1^{\prime}-\right.$ biphenyl]-4-amine (TPA-DEPVI)

A mixture of phenanthrenequinone $(0.416 \mathrm{~g}, 2 \mathrm{mmol}), 4^{\prime}-$ (diphenylamino)biphenyl-4-carbaldehyde (0.698 g, $2 \mathrm{mmol}$ ), (DDMNPI) (1.20 g, $3 \mathrm{mmol})$, ammonium acetate $(1.54 \mathrm{~g}, 20$ $\mathrm{mmol})$ and glacial acetic acid $(25 \mathrm{~mL})$ was refluxed at $120^{\circ} \mathrm{C}$ for $12 \mathrm{~h}$ under a nitrogen atmosphere. ${ }^{23-25}$ The reaction mixture was poured into methanol and the separated white solid was filtered, washed with water and purified by column chromatography using $\mathrm{CH}_{2} \mathrm{Cl}_{2}$ as the eluent. Yield: $52 \%$ Anal. calcd for $\mathrm{C}_{72} \mathrm{H}_{51} \mathrm{~N}_{5} \mathrm{O}_{2}$ : C, 84.90; $\mathrm{H}, 5.01 ; \mathrm{N}, 6.84$. Found: C, 84.78; H, 4.98; N, 6.79. ${ }^{1} \mathrm{H}$ NMR (400 MHz, $\left.\mathrm{CDCl}_{3}\right) \delta 1.86(\mathrm{~s}$, $3 \mathrm{H}), 4.30(\mathrm{~m}, 4 \mathrm{H}), 4.10(\mathrm{~s}, 3 \mathrm{H}), 6.62(\mathrm{~d}, J=16.0 \mathrm{~Hz}, 2 \mathrm{H}), 6.73(\mathrm{~s}$, $3 \mathrm{H}), 6.86(\mathrm{~d}, J=15.0 \mathrm{~Hz}, 1 \mathrm{H}), 7.06-7.23(\mathrm{~m}, 21 \mathrm{H}), 7.48(\mathrm{~d}, J=$ $7.6 \mathrm{~Hz}, 2 \mathrm{H}), 7.62-7.66(\mathrm{~m}, 10 \mathrm{H}), 8.24(\mathrm{~d}, J=8.2 \mathrm{~Hz}, 1 \mathrm{H}), 8.61(\mathrm{t}$, 1H). ${ }^{13} \mathrm{C} \mathrm{NMR}\left(100 \mathrm{MHz}, \mathrm{CDCl}_{3}\right): \delta 20.98,63.21,114.17,123.79$, 123.88, 124.75, 125.74, 126.27, 126.49, 127.37, 128.09, 128.81, 129.08, 129.54, 129.83, 130.04, 130.29, 131.89, 132.06, 134.94, 143.65, 150.17. MALDI-TOF MS: $m / z$. $1017.28\left[\mathrm{M}^{+}\right]$; calcd 1017.34.

\subsection{Device fabrication and measurement}

Devices with the configuration of (i) $[\mathrm{ITO} / \mathrm{NPB}(60 \mathrm{~nm}) / \mathrm{Cz}-$ DPVI/Cz-DMPVI/Cz-DEPVI/TPA-DEPVI $(20 \mathrm{~nm}) / \mathrm{Alq}_{3}(30 \mathrm{~nm}) /$ LiF $(1 \mathrm{~nm}) / \mathrm{Al}(100 \mathrm{~nm})$ ], (ii) hole-only device: [ITO/HATCN (10 nm)/NPB (20 nm)/Cz-DPVI/Cz-DMPVI/Cz-DEPVI/TPADEPVI $(60 \mathrm{~nm}) / \mathrm{NPB}(20 \mathrm{~nm}) / \mathrm{Al}(100 \mathrm{~nm})]$, (iii) electron-only device: [ITO/TPBi (10 nm)/Cz-DPVI/Cz-DMPVI/Cz-DEPVI/TPADEPVI $(60 \mathrm{~nm}) / \mathrm{TPBi}(10 \mathrm{~nm}) / \mathrm{LiF}(1 \mathrm{~nm}) / \mathrm{Al}(100 \mathrm{~nm})]$, (iv) green device: ITO/NPB (40 nm)/TCTA $(5 \mathrm{~nm}) / \mathrm{Cz}-\mathrm{DPVI}(30 \mathrm{~nm})$ : $5 \mathrm{wt} \% \operatorname{Ir}(\mathrm{ppy})_{3} / \mathrm{Cz}-\mathrm{DMPVI}(30 \mathrm{~nm}): 5 \mathrm{wt} \% \operatorname{Ir}(\mathrm{ppy})_{3} / \mathrm{Cz}-\mathrm{DEPVI}$ $(30 \mathrm{~nm}): 5 \mathrm{wt} \% \operatorname{Ir}(\text { ppy })_{3} /$ TPA-DEPVI $(30 \mathrm{~nm}): 5 \mathrm{wt} \% \operatorname{Ir}(\mathrm{ppy})_{3} /$ TPBI $(50 \mathrm{~nm}) / \mathrm{LiF}(1 \mathrm{~nm}) / \mathrm{Al}(100 \mathrm{~nm})]$ and $(\mathrm{v})$ red device: ITO/ NPB (40 nm)/TCTA (5 nm)/Cz-DPVI (30 nm): $8 \mathrm{wt} \% \operatorname{Ir}(\mathrm{MQ})_{2}(-$ acac)/Cz-DMPVI (30 nm): $8 \mathrm{wt} \% \operatorname{Ir}(\mathrm{MQ})_{2}$ (acac)/Cz-DEPVI $(30$ $\mathrm{nm}): \quad 8 \quad \mathrm{wt} \% \quad \operatorname{Ir}(\mathrm{MQ})_{2}(\mathrm{acac}) / \mathrm{TPA}-\mathrm{DEPVI} \quad(30 \quad \mathrm{~nm}): \quad 8 \mathrm{wt} \%$ $\left.\operatorname{Ir}(\mathrm{MQ})_{2}(\mathrm{acac}) / \mathrm{TPBI}(50 \mathrm{~nm}) / \mathrm{LiF} \quad(1 \mathrm{~nm}) / \mathrm{Al}(100 \mathrm{~nm})\right]$ were fabricated on pre-cleaned ITO-coated glass substrates with a resistance of $20 \Omega \mathrm{sq}^{-1}$. Current density-voltage characteristics were measured with a Keithley 2400 power source. EL spectra and CIE coordinates were recorded with a spectrometer (USB-650-VIS-NIR, Ocean Optics, Inc, USA).

\section{Results and discussion}

Efficient blue emitters, namely, 2-(4-(9H-carbazol-9-yl) phenyl)-1-(4-((E)-2-(1-(2,3-dihydrobenzo[b][1,4]dioxin-8-yl)$1 H$-phenanthro[9,10- $d]$ imidazol-2-yl)vinyl)phenyl)- $1 H$-phenanthro[9,10- $d]$ imidazole (Cz-DPVI), 2-(4-(9H-carbazol-9-yl) phenyl)-1-(4-((E)-2-(1-((2,3-dihydrobenzo[ $b][1,4]$ dioxin-6-yl) methyl)-1 $H$-phenanthro[9,10- $d$ ]imidazol-2-yl)vinyl) phenyl)$1 H$-phenanthro $[9,10-d]$ imidazole (Cz-DMPVI), 2-(4-(9Hcarbazol-9-yl)phenyl)-1-(4-((E)-2-(1-(1-(2,3-dihydrobenzo[b] $[1,4]$ dioxin-7-yl)ethyl)-1 $H$-phenanthro[9,10- $d]$ imidazol-2-yl) vinyl)phenyl)-1 $H$-phenanthro[9,10- $d]$ imidazole (Cz-DEPVI) and $\quad 4^{\prime}-(1-(4-(2-(1-(2,3-$ dihydrobenzo $[b][1,4]$ dioxin-6-yl)-1Hphenanthro[9,10- $d]$ imidazol-2-yl)vinyl)phenyl)- $1 H$-phenanthro[9,10- $d]$ imidazol-2-yl)- $N, N$-diphenyl-[1,1'-biphenyl]-4amine (TPA-DEPVI) were synthesized in appreciable yields of $60 \%, 58 \%, 50 \%$ and $52 \%$ and characterized via ${ }^{1} \mathrm{H}$ and ${ }^{13} \mathrm{C}$ NMR, high resolution mass spectroscopy and elemental 
Table 1 Optical and thermal properties and device performances of Cz-DPVI, Cz-DMPVI, Cz-DEPVI and TPA-DEPVI

\begin{tabular}{|c|c|c|c|c|}
\hline Emitters & Cz-DPVI & Cz-DMPVI & Cz-DEPVI & TPA-DEPVI \\
\hline$\lambda_{\mathrm{ab}}(\mathrm{nm})(\mathrm{sol} / \mathrm{film})$ & $252,320,356 / 323,360$ & $254,328,360 / 330,364$ & $260,331,366 / 334,372$ & $255,332,368 / 335,370$ \\
\hline$\lambda_{\mathrm{em}}(\mathrm{nm})(\mathrm{sol} / \mathrm{film})$ & $390,408 / 412,420$ & $399,415 / 420,432$ & $401,428 / 410,440$ & $409,432 / 415,449$ \\
\hline$T_{\mathrm{g}} / T_{\mathrm{d} 5}\left({ }^{\circ} \mathrm{C}\right)$ & $163 / 521$ & $174 / 546$ & $195 / 566$ & $190 / 558$ \\
\hline$\phi($ soln/film $)$ & $0.76 / 0.83$ & $0.80 / 0.89$ & $0.82 / 0.91$ & $0.78 / 0.86$ \\
\hline HOMO/LUMO (eV) & $-5.60 /-2.58$ & $-5.59 /-2.61$ & $-5.46 /-2.70$ & $-5.50 /-2.65$ \\
\hline$E_{\mathrm{g}}(\mathrm{eV})$ & 3.02 & 2.93 & 2.76 & 2.85 \\
\hline$E_{\mathrm{S}} / E_{\mathrm{T}}(\mathrm{eV})$ & $2.62 / 2.34$ & $2.74 / 2.40$ & $2.88 / 2.49$ & $2.80 / 2.46$ \\
\hline$\tau(\mathrm{ns})$ & 1.83 & 1.92 & 2.32 & 2.10 \\
\hline$k_{\mathrm{r}} \times 10^{8}\left(\mathrm{~s}^{-1}\right)$ & 4.15 & 4.17 & 3.53 & 3.71 \\
\hline$k_{\mathrm{nr}} \times 10^{8}\left(\mathrm{~s}^{-1}\right)$ & 1.31 & 1.03 & 0.78 & 1.05 \\
\hline$V_{1000}(\mathrm{~V})$ & 3.4 & 3.2 & 2.8 & 2.9 \\
\hline$L\left(\mathrm{~cd} \mathrm{~m}^{-2}\right)$ & 13629 & 13841 & 13955 & 13856 \\
\hline$\eta_{\mathrm{ex}}(\%)$ & 4.6 & 4.9 & 6.9 & 5.7 \\
\hline$\eta_{\mathrm{c}}\left(\mathrm{cd} \mathrm{A}^{-1}\right)$ & 4.9 & 5.4 & 6.0 & 5.7 \\
\hline$\eta_{\mathrm{p}}\left(\operatorname{lm} \mathrm{W}^{-1}\right)$ & 4.3 & 5.0 & 5.4 & 5.2 \\
\hline $\operatorname{CIE}(x, y)$ & $0.15,0.08$ & $0.15,0.08$ & $0.15,0.06$ & $0.15,0.07$ \\
\hline $\mathrm{EL}(\mathrm{nm})$ & 419 & 430 & 439 & 442 \\
\hline$\eta_{\mathrm{IOE}}(\%)^{\mathrm{a}}$ & 22.0 & 23.0 & 24.5 & 23.5 \\
\hline$\eta_{\mathrm{S}}$ & 26.5 & 25.8 & 26.9 & 27.3 \\
\hline
\end{tabular}
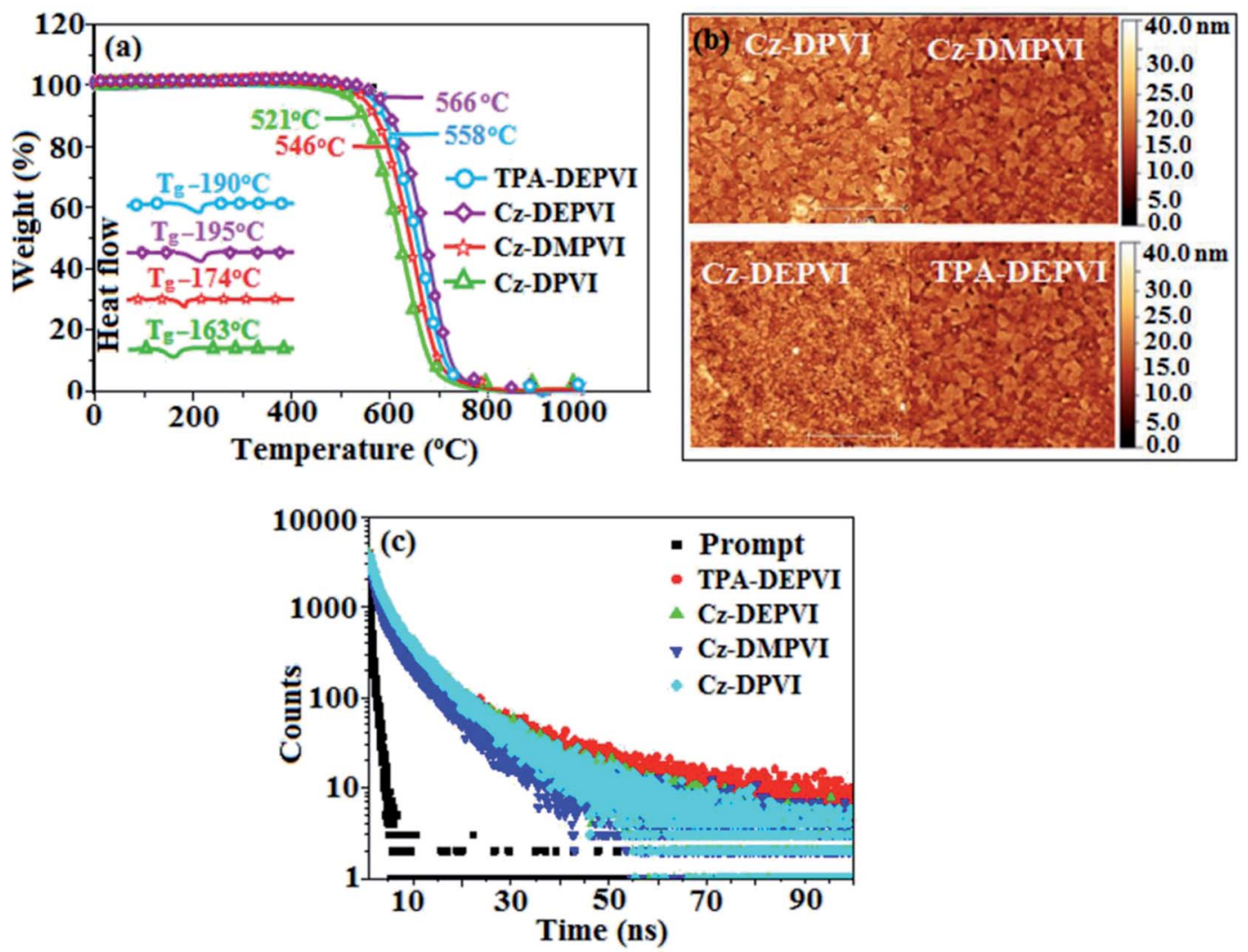

Fig. 1 (a) TGA (inset: DSC) graph, (b) AFM images and (c) lifetime spectra of Cz-DPVI, Cz-DMPVI, Cz-DEPVI and TPA-DEPVI.

analysis. The synthetic route for the emissive materials is displayed in Scheme 2. The weak electron-donating carbazole in Cz-DPVI, Cz-DMPVI and Cz-DEPVI increased the \% LE component in the emissive state $\left(\mathrm{S}_{1}\right.$ HLCT $)$, which resulted in a high photoluminance efficiency $\left(\eta_{\mathrm{PL}}\right)$.
3.1. Potential energy surface (PES) scan studies and thermal properties

The effect of the configuration of the emissive materials on the photophysical properties of Cz-DPVI, Cz-DMPVI, CzDEPVI and TPA-DEPVI was investigated theoretically using 

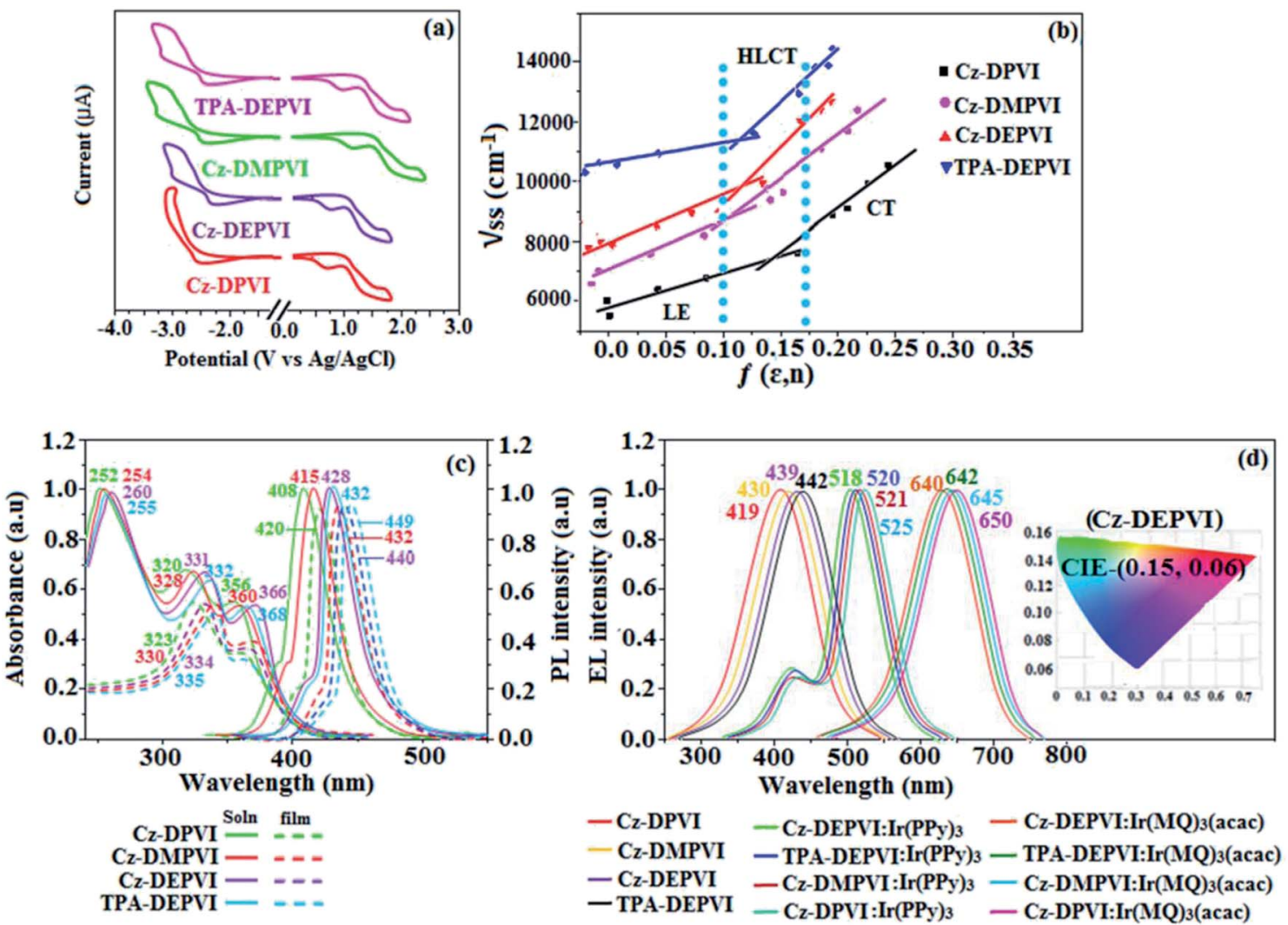

Fig. 2 (a) Cyclic voltammograms, (b) Lippert-Mataga plot, (c) normalized absorption and emission spectra and (d) EL spectra (inset: CIE coordinates) of Cz-DPVI, Cz-DMPVI, Cz-DEPVI and TPA-DEPVI.

DFT/B3LYP/6-31G (d) level (Fig. S1†). A potential energy surface scan for C67-C109-C58-C57 (Cz-DPVI)/C67-C109C58-C57 (Cz-DMPVI)/C67-C109-C58-C57 (Cz-DEPVI)/C67C109-C58-C57 (TPA-DEPVI) was performed, where the geometrical parameters were relaxed, and the torsional angles were altered in steps of $0^{\circ}, 20^{\circ}, 40^{\circ}, 60^{\circ} \ldots 360^{\circ}$. The minimum energy conformation in the PES of Cz-DPVI, CzDMPVI, Cz-DEPVI and TPA-DEPVI showed that the side chain (E)-1-(2,3-dihydrobenzo[ $b][1,4]$ dioxin-6-yl)-2-(4-styryl)$1 H$-phenanthro[9,10- $d]$ imidazole (Cz-DPVI)/(E)-1-((2,3-dihydrobenzo $[b][1,4]$ dioxin-6-yl)methyl)-2-styryl- $1 H$-phenanthro [9,10- $d$ ]imidazole (Cz-DMPVI)/(E)-1-(1-(2,3-dihydrobenzo[ $b]$ $[1,4]$ dioxin-6-yl)ethyl)-2-styryl-1 $H$-phenanthro[9,10- $d]$ imidazole (Cz-DEPVI and TPA-DEPVI) was orthogonally attached to the imidazole nitrogen atom (N23) by $\sim 90.0^{\circ}$. The orthogonal dihedral angles $\left(\sim 90.0^{\circ}\right)$ between the side chain at $\mathrm{N}(23)$ and the substituent at main frame $\mathrm{C}(25)$, i.e., the 2(4-(9H-carbazol-9-yl)phenyl)-1-methyl-1H-phenanthro[9,10- $d]$ imidazole core (Cz-DPVI, Cz-DMPVI, Cz-DEPVI and TPADEPVI) $/ N, N$-diphenyl-[1,1'-biphenyl]-4-amine (TPA-DEPVI), reduces the intermolecular packing. Therefore, the side chain at $\mathrm{N}(23)$ and rigid frame at $\mathrm{C}(25)$ act as hole-trapping sites and the peripheral core blocks the electron-trapping sites. Hence, effective carrier injection and transport ability will be expected from the Cz-DPVI, Cz-DMPVI, Cz-DEPVI and TPA-DEPVI emitters. The relative carrier transport ability of the title materials was analysed using hole-only and electron- only devices. The incorporation of the side capping $(E)-1-(2,3-$ dihydrobenzo[ $b][1,4]$ dioxin-6-yl)-2-(4-styryl)- $1 H$-phenanthro $[9,10-d]$ imidazole derivative at $\mathrm{N}(23)$ and $2-(4-(9 H$-carbazol-9yl)phenyl)-1-methyl-1 $H$-phenanthro[9,10- $d]$ imidazole $(\mathrm{Cz}-$ DEPVI) $/ N, N$-diphenyl-[1,1'-biphenyl]-4-amine (TPA-DEPVI) at $\mathrm{C}(25)$ enhanced the degree of molecular distortion and suppressed the formation of aggregation and $\pi-\pi$ stacking in the film state, resulting in an amorphous film (smooth and pinhole-free) during fabrication. ${ }^{26}$ The side capping at $\mathrm{N}(23)$ is twisted about the 2-(4-(9H-carbazol-9-yl)phenyl)-1-methyl$1 H$-phenanthro[9,10- $d]$ imidazole $(\mathrm{Cz}$-DEPVI) $/ N, N$-diphenyl-

[1,1'-biphenyl]-4-amine (TPA-DEPVI) core with a dihedral angle of $90.1^{\circ}$ (Cz-DPVI)/91.0 $(\mathrm{Cz}-\mathrm{DMPVI}) / 92.0^{\circ}$ (Cz-DEPVI)/ $90.9^{\circ}$ (TPA-DMPVI)/and 9-( $p$-tolyl)-9H-carbazole at the imidazole carbon atom (C25) is tilted about the phenanthrimidazole core at an angle of $86.1^{\circ}(\mathrm{Cz}-\mathrm{DPVI}) / 87.2^{\circ}(\mathrm{Cz}-$ DMPVI $/ 87.9^{\circ}$ (Cz-DEPVI)/85.3 ${ }^{\circ} \mathrm{N}, \mathrm{N}$-diphenyl-[1,1'-biphenyl]4-amine (TPA-DEPVI). These orthogonal dihedral angles confirmed the non-coplanar twisting conformation of $\mathrm{Cz}^{-}$ DPVI, Cz-DMPVI, Cz-DEPVI and TPA-DEPVI, ${ }^{27-29}$ which suppresses the red shift and harvests high quantum efficiency $\left(\eta_{\mathrm{ex}}\right)$ in the film state by restraining intermolecular interaction. ${ }^{30}$ The incorporation of a highly rigid bulky moiety at $\mathrm{C}(25)$ and side capping at $\mathrm{N}(23)$ enlarged their size $\left(T_{\mathrm{d} 5}\right.$ and $\left.T_{\mathrm{g}}\right)$ and improved their thermal stability. The blue emissive phenanthroimidazole with maximum thermal stability is required for efficient devices (Table 1). The glass 
Table 2 Computed [zindo (singlet or triplet, $n$ states $=10)$ ] singlet $\left(E_{S}\right)$ and triplet $\left(E_{T}\right)$ energies, oscillator strength $(f)$, dipole moment $(\mu)$ and singlet-triplet energy difference $\left(\Delta E_{S-T}\right)$ of TPA-DEPVI from NTOs

\begin{tabular}{|c|c|c|c|c|c|c|c|}
\hline Energy level & $E_{\mathrm{S}}(\mathrm{eV})$ & Oscillator strength $(f)$ & $\mu(\mathrm{D})$ & NTO Transitions & $E_{\mathrm{T}}(\mathrm{eV})$ & $\Delta E_{\mathrm{S}-\mathrm{T}}(\mathrm{eV})$ & NTO Transitions \\
\hline 1 & 0.57 & 0.3108 & 2.3747 & $181 \stackrel{74 \%}{\longrightarrow} 182$ & 0.25 & 0.32 & $182 \stackrel{57 \%}{\longrightarrow} 202$ \\
\hline 3 & 1.31 & 0.0740 & 0.8996 & $181 \stackrel{34 \%}{\longrightarrow} 183$ & 1.61 & 0.30 & $182 \stackrel{47 \%}{\longrightarrow} 185$ \\
\hline 4 & 1.93 & 0.1036 & 2.3864 & $181 \stackrel{38 \%}{\longrightarrow} 195$ & 1.86 & 0.07 & $182 \stackrel{33 \%}{\longrightarrow} 183$ \\
\hline 5 & 2.12 & 0.0693 & 1.0767 & $181 \stackrel{31 \%}{\longrightarrow} 184$ & 2.01 & 0.11 & $179 \stackrel{24 \%}{\longrightarrow} 184$ \\
\hline 7 & 2.58 & 0.0669 & 0.8435 & $181 \stackrel{33 \%}{\longrightarrow} 199$ & 2.13 & 0.45 & $178 \stackrel{18 \%}{\longrightarrow} 183$ \\
\hline 8 & 2.76 & 0.0004 & 0.5623 & $181 \stackrel{51 \%}{\longrightarrow} 186$ & 2.18 & 0.58 & $172 \stackrel{25 \%}{\longrightarrow} 188$ \\
\hline 9 & 2.91 & 0.0012 & 1.2258 & $181 \stackrel{50 \%}{\longrightarrow} 189$ & 2.50 & 0.41 & $172 \stackrel{19 \%}{\longrightarrow} 194$ \\
\hline 10 & 2.99 & 0.0123 & 1.8824 & $181 \stackrel{58 \%}{\longrightarrow} 190$ & 2.60 & 0.39 & $182 \stackrel{22 \%}{\longrightarrow} 184$ \\
\hline
\end{tabular}

transition temperature $\left(T_{\mathrm{g}}\right)$ of $163{ }^{\circ} \mathrm{C}, 174{ }^{\circ} \mathrm{C}, 195{ }^{\circ} \mathrm{C}$, and $190{ }^{\circ} \mathrm{C}$ was observed for Cz-DPVI, Cz-DMPVI, Cz-DEPVI and TPA-DEPVI, respectively (Fig. 1). The side capping (E)-1-(2,3dihydrobenzo[ $b][1,4]$ dioxin-6-yl)-2-(4-styryl)- $1 H$-phenanthro $[9,10-d]$ imidazole derivatives at $\mathrm{N}(23)$ increased the glass transition temperature compared with phenyl substitution at the imidazole nitrogen. ${ }^{31}$ The decomposition temperature $\left(T_{\mathrm{d} 5}\right)$ of compounds Cz-DPVI, Cz-DMPVI, Cz-DEPVI and TPADEPVI were measured to be $521{ }^{\circ} \mathrm{C}, 546^{\circ} \mathrm{C}, 566^{\circ} \mathrm{C}$ and $558^{\circ} \mathrm{C}$, respectively (Fig. 1). The high $T_{\mathrm{d} 5}$ of all four compounds indicates the high resistance of their fused aromatic rings to thermolysis, and their high $T_{\mathrm{g}}$ and $T_{\mathrm{d} 5}$ could enhance the device lifetime (Fig. 1) and expected to form a stable film during device fabrication. ${ }^{32-34}$ The thermal morphological stability of the Cz-DPVI, Cz-DMPVI, Cz-DEPVI and TPA-DEPVI thin films were examined via atomic force microscopy (AFM) measurement at room temperature and also at $100{ }^{\circ} \mathrm{C}$ for $10 \mathrm{~h}$. The root-mean-square roughness (RMS) of the Cz-DPVI $(0.24 \mathrm{~nm})$, Cz-DMPVI $(0.28 \mathrm{~nm})$, Cz-DEPVI $(0.32 \mathrm{~nm})$ and TPADEPVI $(0.39 \mathrm{~nm})$ thin-film surfaces showed that there were no substantial changes before and after annealing $\left(100^{\circ} \mathrm{C}, 10 \mathrm{~h}\right)$ (Fig. 1), which also supports the suitability of these emissive materials for the fabrication of blue OLEDs.

The HOMO orbital is distributed over the 2-(4- $(9 H$-carbazol-9-yl)phenyl)-1 $H$-phenanthro[9,10- $d]$ imidazole fragment (Cz-DPVI, Cz-DMPVI and Cz-DEPVI)/4'-(1H-phenanthro[9,10- $d$ ]imidazol-2-yl)- $N, N$-diphenyl-[1,1'-biphenyl]-4-amine fragment (TPA-DEPVI); whereas, the LUMO orbital localized on the (E)-1-(2,3-dihydrobenzo[b][1,4]dioxin-6-yl)-2-(4-styryl)- $1 H^{-}$ phenanthro[9,10- $d]$ imidazole derivatives attached to the nitrogen atom N(23) (Fig. S2 $\dagger$ ). The HOMO and LUMO of CzDPVI, Cz-DMPVI, Cz-DEPVI and TPA-DEPVI display adequate separation features and their differences are quite small, which benefits the hole- and electron-transport properties (bipolar properties) and reduces the singlet-triplet splitting $\left(\Delta_{\mathrm{ST}}\right) .^{35}$ Hence, the HOMO and LUMO moieties individually undertake electron and hole transport functions. The calculated electron/hole transfer integrals for Cz-DEPVI of 0.029 / $0.052 \mathrm{eV}, \mathrm{Cz}$-DPVI of 0.024/0.44 eV, Cz-DMPVI of 0.26/0.50 eV and TPA-DMPVI of $0.23 / 0.55 \mathrm{eV}$ reveal that these materials are bipolar materials. Moreover, these compounds show both reduction and oxidation waves, and thus, should have good electron and hole transport abilities (Fig. 2).

\subsection{Photophysical properties}

The photophysical properties of Cz-DPVI, Cz-DMPVI, Cz-DEPVI and TPA-DEPVI were investigated via absorption $\left(\lambda_{\mathrm{abs}}\right)$ and emission $\left(\lambda_{\text {emi }}\right)$ spectroscopy. The electronic spectra of Cz-DPVI, Cz-DMPVI, Cz-DEPVI and TPA-DEPVI were recorded in $\mathrm{CH}_{2} \mathrm{Cl}_{2}$ (Fig. 2). Since the core fragment of Cz-DPVI, Cz-DMPVI, CzDEPVI and TPA-DEPVI is same, these new emitters exhibit identical absorptions (solution/film) $252\left(\varepsilon_{\max }=39682 \mathrm{~cm}^{-1}\right.$

Table 3 Electroluminescent efficiencies of the green Cz-DPVI:Ir(PPy) ${ }_{3}, \mathrm{Cz}-\mathrm{DMPVI}: \operatorname{Ir}(\mathrm{PPy})_{3}, \mathrm{Cz}-\mathrm{DEPVI}: \operatorname{Ir}(\mathrm{PPy})_{3}$ and red Cz-DPVI:Ir(MQ) $3(\mathrm{acac})_{\text {, }}$ Cz-DMPVI:Ir(MQ) ${ }_{3}$ (acac) and Cz-DEPVI:Ir(MQ) ${ }_{3}$ (acac)

\begin{tabular}{|c|c|c|c|c|c|c|c|}
\hline Emitters & $V_{1000}(\mathrm{~V})$ & $L\left(\mathrm{~cd} \mathrm{~m}^{-2}\right)$ & $\eta_{\mathrm{ex}}(\%)$ & $\eta_{\mathrm{c}}\left(\mathrm{cd} \mathrm{A}^{-1}\right)$ & $\eta_{\mathrm{p}}\left(\operatorname{lm~\mathrm {W}^{-1})}\right.$ & $\operatorname{CIE}(x, y)$ & $\mathrm{EL}(\mathrm{nm})$ \\
\hline Cz-DPVI:Ir(PPy) ${ }_{3}$ & 3.1 & 7146 & 18.2 & 25.3 & 27.0 & $0.32,0.60$ & 428,525 \\
\hline Cz-DMPVI:Ir(PPy) ${ }_{3}$ & 2.8 & 8056 & 18.9 & 26.8 & 28.9 & $0.31,0.60$ & 430,521 \\
\hline TPA-DEPVI:Ir(PPy) ${ }_{3}$ & 2.7 & 8756 & 19.0 & 27.1 & 29.5 & $0.31,0.60$ & 431,520 \\
\hline Cz-DPVI:Ir(MQ) $)_{3}(\mathrm{acac})$ & 3.0 & 17215 & 17.6 & 21.1 & 25.4 & $0.64,0.37$ & 650 \\
\hline Cz-DMPVI:Ir(MQ) $)_{3}(\mathrm{acac})$ & 2.7 & 20628 & 19.0 & 24.9 & 26.8 & $0.64,0.37$ & 645 \\
\hline
\end{tabular}




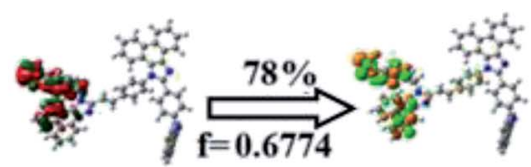

S2

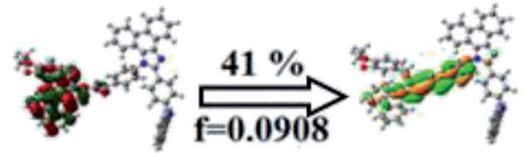

S3

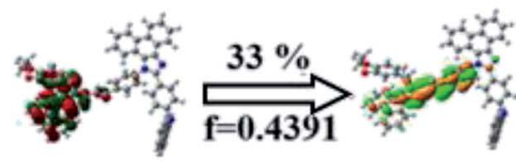

4
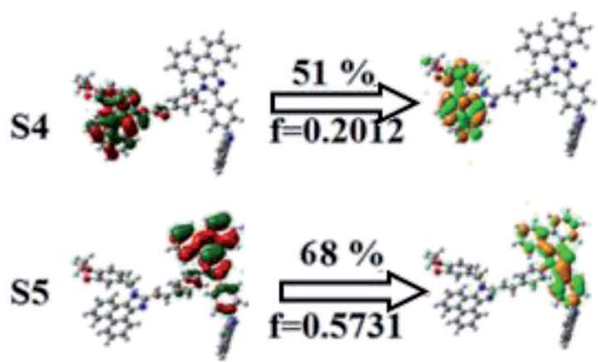

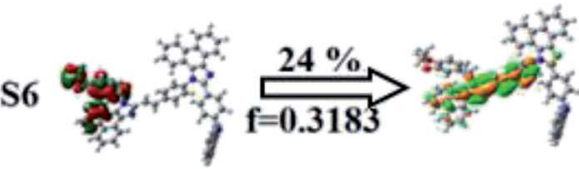
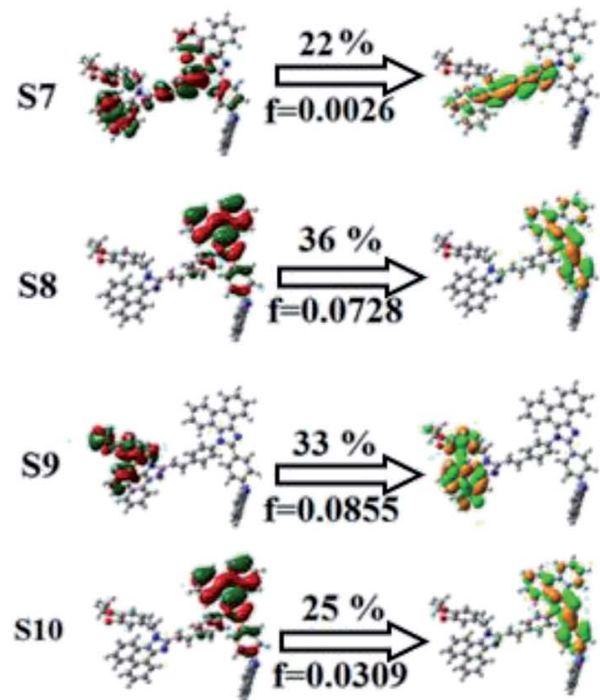

T1

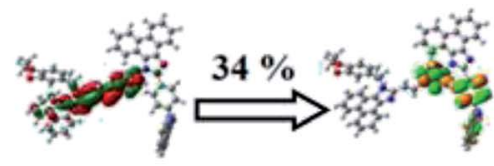

T2

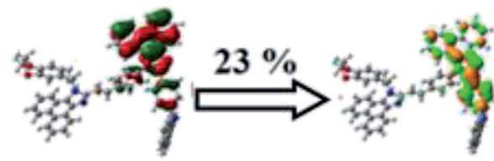

T3

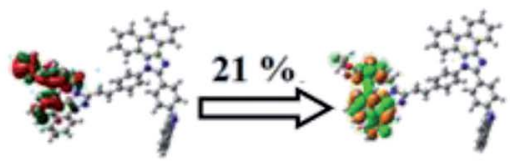

T4

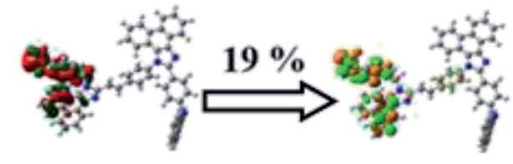

T5

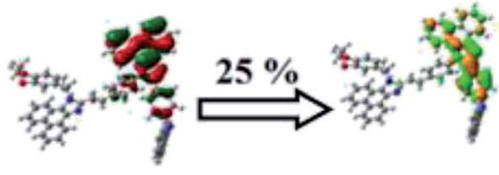

T6

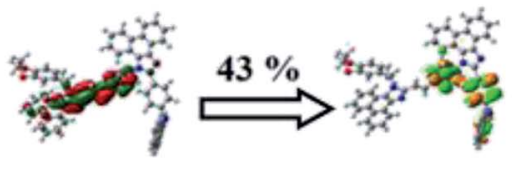

T7

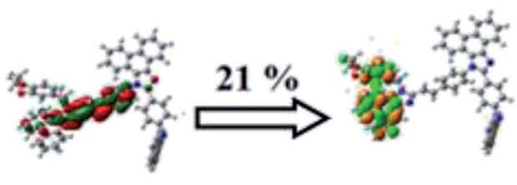

T8

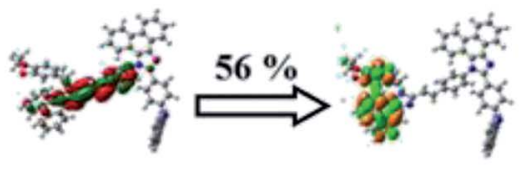

T9

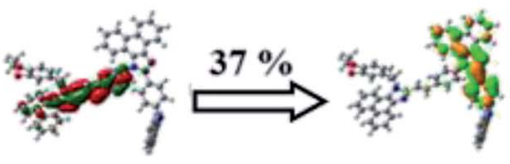

T10

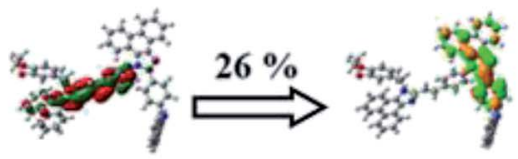

Fig. 3 Natural transition orbital (NTO) pairs with transition character analysis for the singlet states $\left(\mathrm{S}_{1}-\mathrm{S}_{10}\right)$ and triplet states $\left(\mathrm{T}_{1}-\mathrm{T}_{10}\right)$ of $\mathrm{Cz}$-DEPVI [f-oscillator strength and \% weights of hole-particle].

$\left.\mathrm{M}^{-1}\right) / 320,356 / 323,360 \mathrm{~nm}(\mathrm{Cz}-\mathrm{DPVI}), 254\left(\varepsilon_{\max }=39370 \mathrm{~cm}^{-1}\right.$ $\left.\mathrm{M}^{-1}\right)$, 328, 360/330, $364 \mathrm{~nm}$ (Cz-DMPVI), $260 \quad\left(\varepsilon_{\max }=\right.$ $38461 \mathrm{~cm}^{-1} \mathrm{M}^{-1}$ ), 331, 366/334, $372 \mathrm{~nm}$ (Cz-DEPVI) and 255 $\left(\varepsilon_{\max }=39215 \mathrm{~cm}^{-1} \mathrm{M}^{-1}\right.$ ), 332, 368/335, $370 \mathrm{~nm}$ (TPA-DEPVI). The absorption in the range of 328 to $372 \mathrm{~nm}$ is due to intramolecular charge transfer (ICT) from the donor (carbazole/ triphenylamine) to acceptor (phenanthrimidazole) and the absorption at around $248 \mathrm{~nm}$ is attributed to the $\pi-\pi^{*}$ transition. ${ }^{36-38}$ The intramolecular charge transfer (ICT) is further confirmed by MEP (Fig. S1†). Compared with solution, the small red shift in the corresponding films reveals that suppressed $\pi-$ $\pi^{*}$ stacking exists in the solid state. ${ }^{39}$ The observed larger red shift supports the charge-transfer (CT) in the twisted geometry of Cz-DPVI, Cz-DMPVI, Cz-DEPVI and TPA-DEPVI. The Cz-DPVI, Cz-DMPVI, Cz-DEPVI and TPA-DEPVI derivatives show blue emission at 408, 415, 428 and $432 \mathrm{~nm}$, respectively, in $\mathrm{CH}_{2} \mathrm{Cl}_{2}$ (Fig. 2) and their emission peak is red shifted as the polarity of the solvent increases (Fig. S3†), which is likely due to 
a polarization-induced spectral shift. ${ }^{40}$ Compared with TPADEPVI, Cz-DEPVI exhibits a higher blue shift in absorption and emission, which is attributed to the poor electron donor ability of $\mathrm{Cz}$ relative to TPA. The increased LE composition with a decrease in CT in the $\mathrm{S}_{1}$ emissive state is likely to be the reason for this blue shift. The full width at half maximum in the absorption spectrum of Cz-DEPVI $(43 \mathrm{~nm})$ is narrower than to that of TPA-DEPVI (60 nm) (Fig. 2). This observation indicates a decrease in CT component in Cz-DEPVI in the $\mathrm{S}_{1}$ state, which is in good agreement with the NTO description for the $S_{0} \rightarrow S_{1}$ transition. The emission peak of TPA-DEPVI and Cz-DEPVI exhibits a blue-shift relative to their parent compounds, which is contrary to the general observation, i.e., the extension of $\pi$-conjugation leads to a red-shifted emission. ${ }^{\mathbf{4 1}}$ The enhanced LE component is equivalent to the suppressed CT component in the Cz-DEPVI emissive state, which results in a blue shift. The increased LE composition is expected to result in a red-shifted PL spectrum; whereas, suppressed CT results in a blue-shifted PL spectrum. From the experimental observation it is known that the latter factor is more dominant than the former. In addition, there is an overlap between the UV and PL spectra of both TPA-DEPVI and Cz-DEPVI due to the enhanced LE character in TPA-DEPVI and Cz-DEPVI than that in their respective parent compounds. Cz-DEPVI exhibits a solvatochromic red shift $(38 \mathrm{~nm})$, which is smaller than that for TPA-DEPVI (68 nm) (Fig. S3, Tables S1 and S2 $\dagger$ ). Similarly, a small absorption shift of $18 \mathrm{~nm}$ and $27 \mathrm{~nm}$ was observed for
Cz-DEPVI and TPA-DEPVI, respectively (Fig. S4, Tables S1 and $\mathrm{S} 2 \dagger)$. These solvatochromic shifts confirmed that the low-lying $\mathrm{S}_{1}$ excited state of TPA-DEPVI and Cz-DEPVI must possess CT character. ${ }^{42-44}$ The \% CT character of the $\mathrm{S} 1$ state in Cz-DEPVI is lower than that in TPA-DEPVI; whereas, the \% LE character in Cz-DEPVI is higher than that in TPA-DEPVI (Table S3†). The solvatochromic effect using the Lippert-Mataga plot is displayed in Fig. 2 (Tables S1 and S2 $\dagger$ ). When the solvent polarity increased, the blue emitters exhibited a larger red shift, which supports the presence of charge transfer (CT) in these molecules. ${ }^{42}$ From the Stokes shift versus solvent polarity function plot, the ground state dipole moment $\left(\mu_{\mathrm{g}}\right)$ can be calculated as follows: $h c\left(\tilde{v}_{\mathrm{abs}}-\tilde{v}_{\mathrm{flu}}\right)=h c\left(h c \tilde{v}_{\mathrm{abs}}^{\mathrm{vac}}-h c \tilde{v}_{\mathrm{flu}}^{\mathrm{vac}}\right)+2\left(\mu_{\mathrm{e}}-\mu_{\mathrm{g}}\right)^{2} / a_{\mathrm{o}}{ }^{3}[(\varepsilon-$ $\left.1 / 2 \varepsilon+1)-1 / 2\left(n^{2}-1 / 2 n^{2}+1\right)\right]$, where $\mu_{\mathrm{g}}$ and $\mu_{\mathrm{e}}$ represent the ground state and excited state dipole moment, $\tilde{v}_{\text {abs }}$ and $\tilde{v}_{\mathrm{abs}}^{\mathrm{vac}}$ the solvent-equilibrated absorption maxima $\left(\lambda_{\text {abs }}\right)$ and that extrapolated to the gas phase, $\tilde{v}_{\text {flu }}$ and $\tilde{v}_{\text {flu }}^{\text {vac }}$ the solvent-equilibrated fluorescence maxima $\left(\lambda_{\text {emi }}\right)$ and that extrapolated to the gas phase, $a_{\mathrm{o}}$ the Onsager cavity and $\varepsilon$ and $n$ the solvent dielectric constant and refractive index, respectively. The non-linear correlation of the Stokes shift $v s$. solvent polarity function plot revealed that there is a transformation in the fitted line between ethyl ether and methylene chloride, where the non-linear correlation supports the presence of both the locally excited state (LE) and charge transfer excited state (CT). The ground state dipole $\left(\mu_{\mathrm{g}}\right)$ of the blue-emitting materials, Cz-DPVI, CzDMPVI, Cz-DEPVI and TPA-DEPVI, was estimated from the

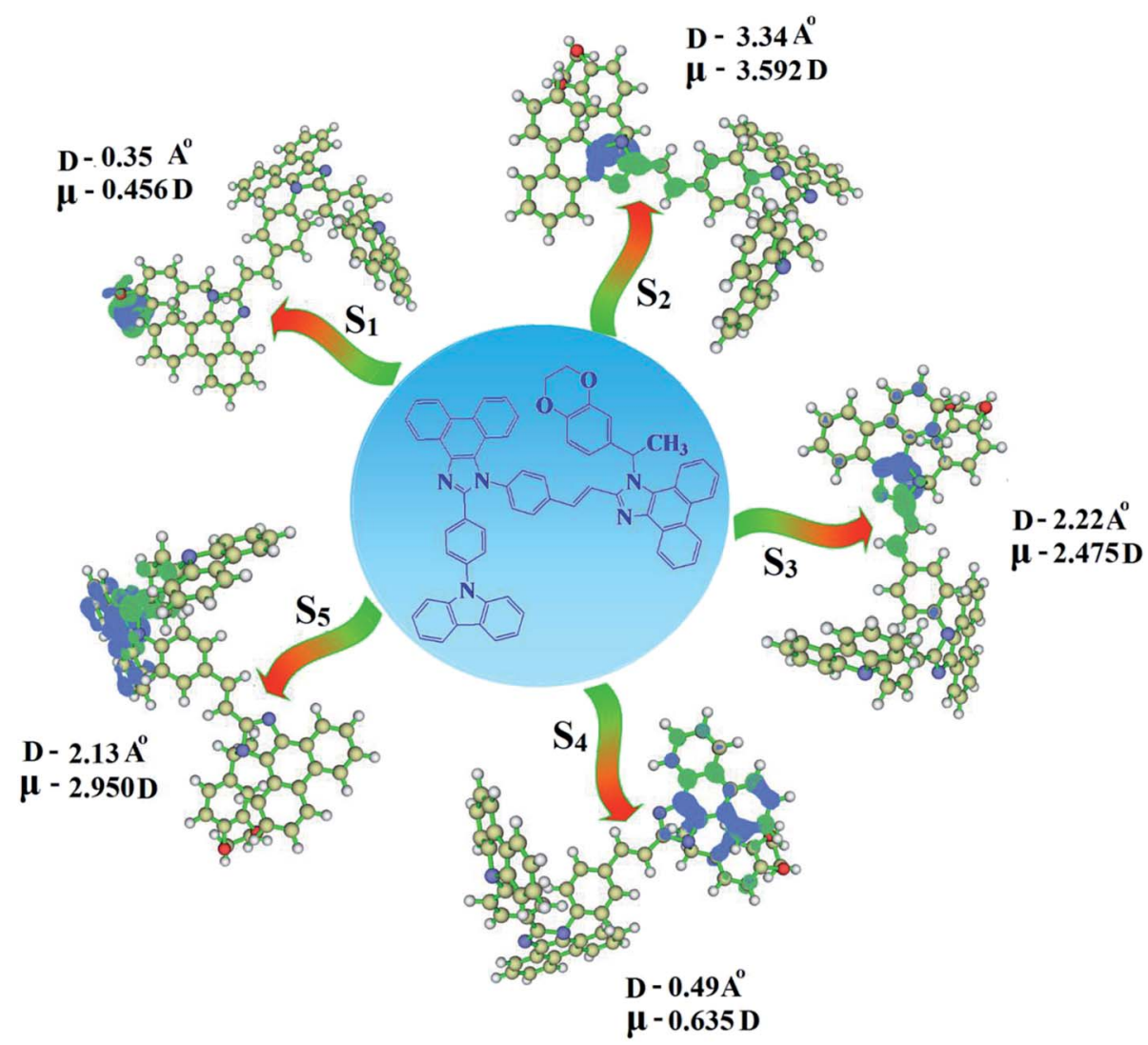

Fig. 4 Hole and particle distribution in $\mathrm{Cz}-\mathrm{DEPVI}\left[\mathrm{S}_{1}-\mathrm{S}_{5}\right.$ states, green increasing electron density and blue decreasing electron density (density $=$ transition $=n \operatorname{IOp}(6 / 8=3))]$. 
density functional theory (DFT) calculations to be 4.8, 5.0, 5.5 and $7.02 \mathrm{D}$, respectively, which is attributed to the local exciton (LE) transition and their calculated $\mu_{\mathrm{e}}$ in highly polar solvents is 22.8, 23.6, 25.8 and $30.8 \mathrm{D}$, respectively. ${ }^{42}$ The large $\mu_{\mathrm{e}}$ in highly polar solvents $(22.8,23.6,25.8$ and $30.8 \mathrm{D})$ is close to that of the charge-transfer molecule 4 -( $N, N$-dimethylamino)-benzonitrile $\left(23.0\right.$ D). ${ }^{44}$ All these results show that CT dominates in more polar media and LE dominates in low polar media, and there is mixed contribution of LE and CT in medium polar solvents (ethyl ether and methylene chloride). ${ }^{10}$ The oscillator strengths of Cz-DPVI, Cz-DMPVI, Cz-DEPVI and TPA-DEPVI are displayed in Tables $\mathrm{S} 4 \dagger$ and 2 . The oscillator strength of the $\mathrm{S}_{1}$ state of $\mathrm{Cz}$ DEPVI (0.6774, Table S4 $\dagger$ ) is higher than that of TPA-DEPVI $(0.3108$, Table 2$)$, which results in higher PL efficiency $\left(\eta_{\mathrm{PL}}\right)$ for Cz-DEPVI. Molecular modification from TPA to Cz caused an increase in the \% LE in the $S_{1}$ emissive state and enhanced the $\eta_{\mathrm{PL}}$ of Cz-DEPVI. To supplement the experimental results, theoretical calculations (NTO) were performed to describe the excited state characteristics of the Cz-DEPVI (Fig. 3) and TPADEPVI (Fig. S5†) materials.

The overlap density between the hole and particle depends on the configuration of the donor-acceptor architecture and the magnitude of the overlap intensity tunes the \% LE and \% CT in the $S_{1}$ state (Fig. S6: $\uparrow$ TPA-DEPVI and Fig. 4: Cz-DEPVI).

The NTO of the $\mathrm{S}_{1}$ and $\mathrm{S}_{2}$ excited states of Cz-DEPVI and TPADEPVI exhibit a hybrid splitting state character, which is derived from the interstate coupling of the LE and CT levels (Tables $\mathrm{S} 4 \dagger$ and 2). The hole contours on the $\mathrm{Cz}$ and TPA moieties are in the opposite phase between the $S_{1}$ and $S_{2}$ states; whereas, the particle on the phenanthrimidazole moiety is same between the $S_{1}$ and $S_{2}$ states for Cz-DEPVI and TPA-DEPVI, respectively. This implies that the interstate hybridization coupling occurs through the positive and negative linear combination between the LE and CT state wavefunction: $\Psi_{\mathrm{S}_{1} / \mathrm{S}_{2}}$ $=c_{\mathrm{LE}} \Psi_{\mathrm{LE}} \pm c_{\mathrm{CT}} \Psi_{\mathrm{CT}}$. The percentage of pure $\mathrm{CT}$ level of $\mathrm{Cz}-$ DEPVI is less than that of TPA-DEPVI as a result of the weaker donor ability of $\mathrm{Cz}$ than TPA, leading to the LE-dominated $\mathrm{S}_{1}$ state in Cz-DEPVI $(\mathrm{LE} \sim 30 \%)$ and LE/CT-balanced $\mathrm{S}_{1}$ state in TPA-DEPVI (LE 20\%). As a result, Cz-DEPVI should exhibit higher photoluminance efficiency $\left(\eta_{\mathrm{PL}}\right)$ and a blue-shifted emission relative to TPA-DEPVI. The singlet state energies were estimated from the geometry of the $S_{0}$ state. The excited state singlet and triplet energies were calculated using the fluorescence and phosphorescence spectra of Cz-DEPVI and TPA-DEPVI at low temperature. The maximum absorption at low temperature was $335 \mathrm{~nm}$ for Cz-DEPVI and $444.1 \mathrm{~nm}$ for TPA-DEPVI and isoenergetic vibronic emission maximum absorption was $336 \mathrm{~nm}$ for Cz-DEPVI and $446.0 \mathrm{~nm}$ for TPADEPVI) in the low-temperature phosphorescence spectrum. A large energy gap occurred between $\mathrm{T}_{1}$ and $\mathrm{T}_{2}$ for both Cz-DEPVI $(1.21 \mathrm{eV})$ and TPA-DEPVI $(0.32 \mathrm{eV})$, originating from their same phenanthrimidazole acceptor group, and the energy gap between $\mathrm{T}_{1}$ and $\mathrm{T}_{2}$ for Cz-DEPVI is larger than that for TPADEPVI (Fig. 5). ${ }^{45,46}$ A very small $\Delta E_{\mathrm{ST}} \approx 0$ was observed between the $S_{1}$ and $T_{2}$ states, facilitating the RISC $\left(T_{2} \rightarrow S_{1}\right)$ process in both Cz-DEPVI (Table S5 $\dagger$ ) and TPA-DEPVI (Table $\mathrm{S} 6 \dagger$ ) as a result of their HLCT state character (Fig. 5). Thus,

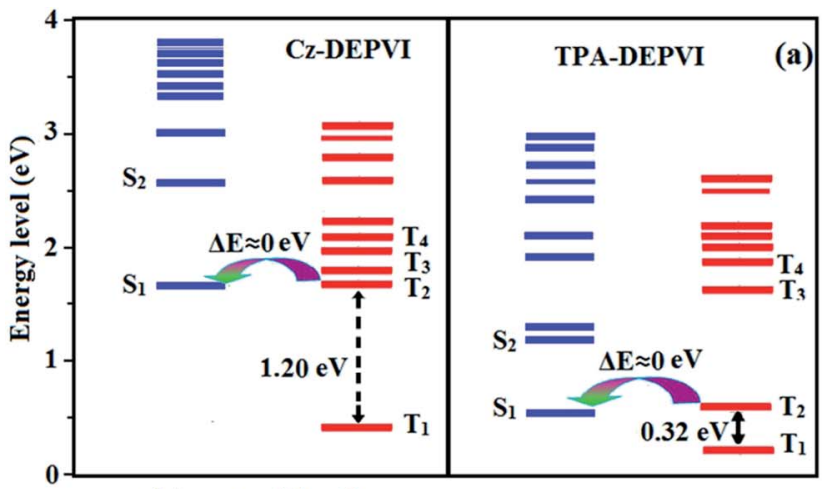

$\mathbf{e}^{-}: h^{+}$recombination

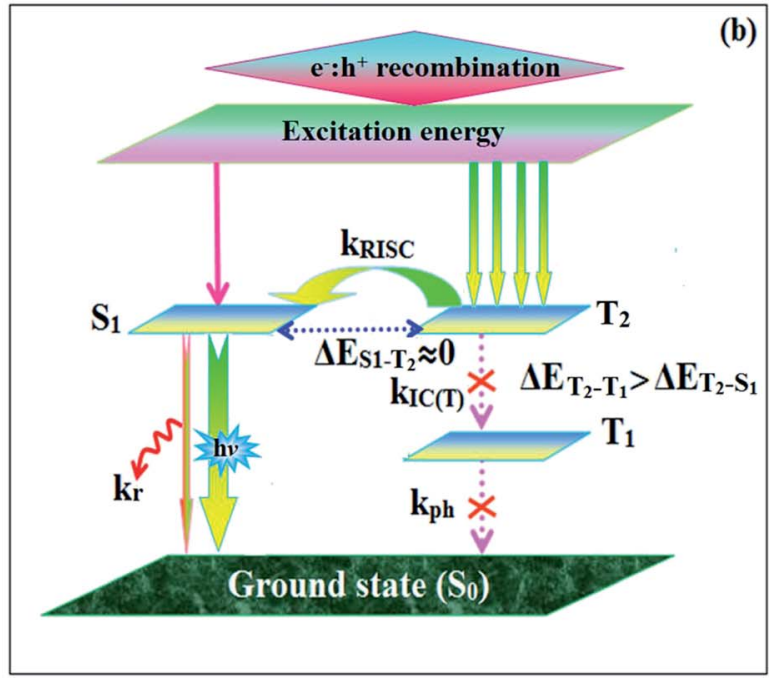

Fig. 5 (a) Energy level of the singlet (S) and triplet (T) states of CzDEPVI and TPA-DEPVI and (b) schematic of the exciton decay process after hole and electron recombination in OLEDs.

compared with TPA-DEPVI, Cz-DEPVI can be expected to exhibit high photoluminance efficiency $\left(\eta_{\mathrm{PL}}\right)$ and high exciton utilisation efficiency $\left(\eta_{\mathrm{S}}\right)$ and further enhance the external quantum efficiency $\left(\eta_{\mathrm{EQE}}\right)$ of fluorescent OLEDs as a result of the increased LE component in its $S_{1}$ state. The formation of a single emissive state can be analysed though the excitation energies of the LE and CT states (Tables S5 and S6 $†$ ).

A similar hole-electron wavefunction between $S_{1}$ and $S_{2}$ was observed in both Cz-DEPVI and TPA-DEPVI, which indicates a quasi-equivalent hybridization between the LE and CT states as a result of their almost isoenergies for the initial LE and CT states (Fig. 6). Therefore, the degree of hybridization between the LE and CT states depends not only on the initial $E_{\mathrm{LE}}-E_{\mathrm{CT}}$ energy gap but also on their interstate coupling strength. ${ }^{47}$ Compared with non-equivalent hybridization, quasi-equivalent hybridization is expected to achieve the combination of high $\eta_{\mathrm{PL}}$ and high $\eta_{\mathrm{S}}$ to maximize the EL efficiency of fluorescent OLED materials due to more balanced LE and CT components in the HLCT state of Cz-DEPVI and TPA-DEPVI. In Cz-DEPVI and TPADEPVI, the LE state is more stabilized than the CT state and the energy gap $\left(E_{\mathrm{S}_{2}}-E_{\mathrm{S}_{1}}\right)$ is small when compared with that of their parent compounds, which result in quasi hybridization. In the case of Cz-DEPVI, the energy gap $\left(E_{\mathrm{S}_{2}}-E_{\mathrm{S}_{1}}\right)$ is reduced, which 

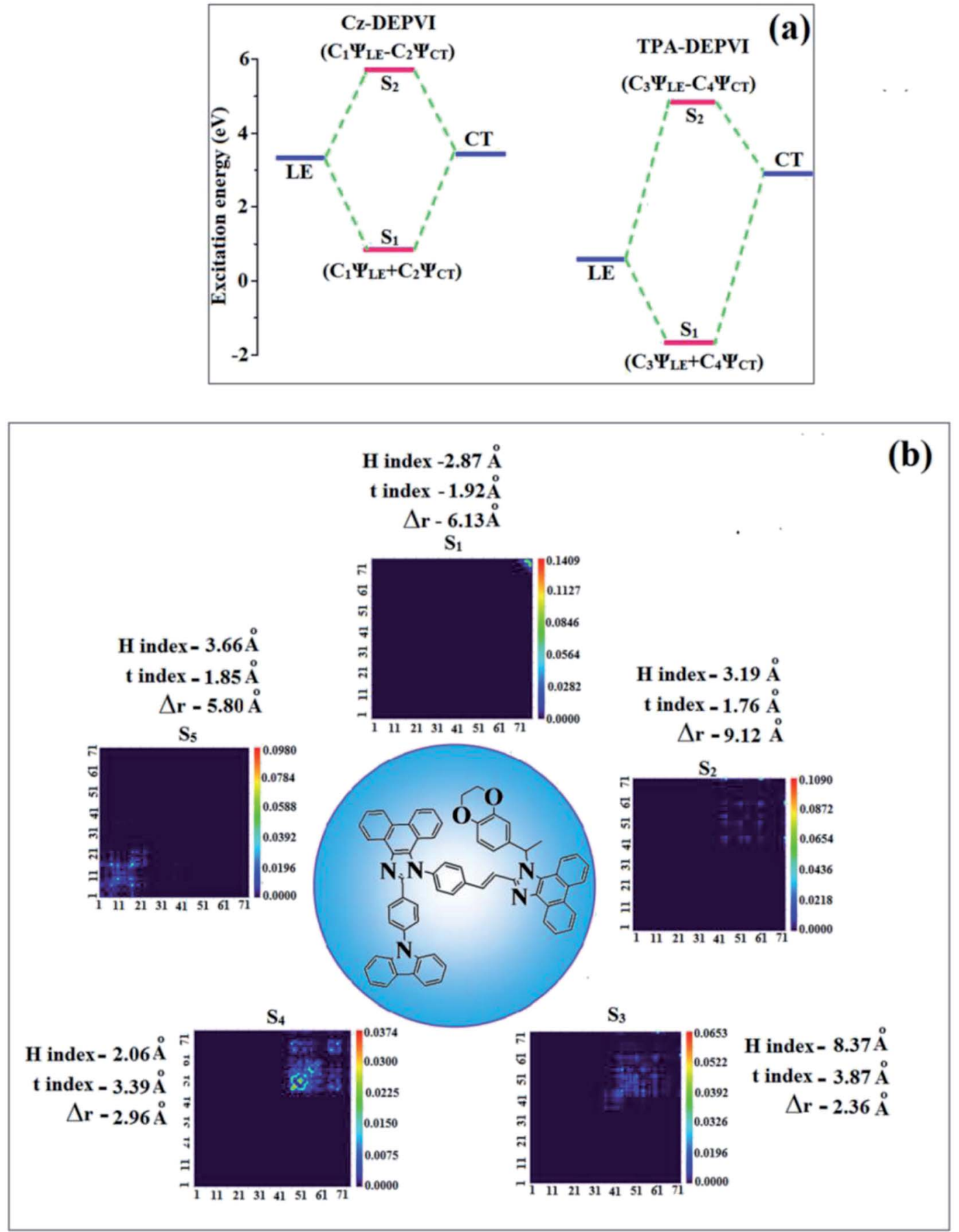

Fig. 6 (a) Schematic diagram of the hybridization processes of the LE and CT states of Cz-DEPVI and TPA-DEPVI and (b) computed contour plots of the transition density matrices (TDM) of Cz-DEPVI for $\left[S_{1}-S_{5}\right.$ states: density $=$ transition $\left.=n / 1 O p(6 / 8=3)\right]$.

results in effective hybridization and improves the OLED efficiency. The composition of the HLCT can be discussed using the wavefunction of the electron-hole pair transition density matrix (TDM), which was plotted in a two-dimension color-filled map (Fig. 6: Cz-DEPVI and Fig. S7: TPA-DEPVI $\dagger$ ). The axes represent the atom in a molecule, which is proportional to the probability of finding an electron and hole in the atomic orbital located on a non-hydrogen atom. Specifically, the diagonal represents the LE component localized on the main backbone, while the off-diagonal region shows the CT component. The qualitatively calculated percentages of LE and CT in the $\mathrm{S}_{1}-\mathrm{S}_{10}$ and $\mathrm{T}_{1}-\mathrm{T}_{10}$ states are displayed in Table $\mathrm{S} 3 . \dagger$ This also supports that HLCT state contributes to hybridization besides the LE and CT states. Upon excitation, an electron is transferred from the donor and localized on the acceptor. Depending on the intramolecular geometrical and electronic coupling, the transferred electron is delocalized from the region of the nearby donor molecule to the vicinity of the acceptor. This effect can be qualitatively studied by analyzing the electron density distribution in the ground and excited states. The computed 
electron-hole properties, distance between hole and electron, transition density, $H$ and $t$ indies and RMSD of an electron and hole for Cz-DEPVI and TPA-DEPVI are displayed in Tables S7 and $\mathrm{S} 8 . \dagger$

The integral value for the holes and electrons in Cz-DEPVI (Table S9 $\dagger$ ) is less than that for TPA-DEPVI (Table S10 $\dagger$ ) with the transition density. The integral overlap of the hole-electron distribution $(S)$ is a measure of the spatial separation of holes and electrons. The integral overlap $(S)$ of holes and electrons and the distance $(D)$ between the centroids of the holes and electrons evidence the existence of LE and CT states (Tables S9 and S10 $\dagger$ ). Compared with their parent compounds, Cz-DEPVI and TPA-DEPVI have small $D$ and high $S$ values; however, the small $D$ and high $S$ of TPA-DEPVI in comparison with Cz-DEPVI indicate the charge transfer (CT) is higher for the TPA-DEPVI isomer. The variation in dipole moment with respect to $S_{0}$ state was also plotted, which was directly evaluated based on the position of the centroid of the holes and electrons. The RMSD of the holes and electrons characterizes the extent of their distribution, were the RMSD of both electrons and holes in Cz-DEPVI (Table $\mathrm{S7} \dagger$ ) is higher in the $X$ direction, which indicates the electron and hole distribution is much broader in the $X$ direction. In contrast, the RMSD of the electrons in TPA-DEPVI
(Table S8 $\dagger$ ) is smaller and that of the holes is higher than $\mathrm{Cz}^{-}$ DEPVI. The $H$ index (half the sum of the axis of the anisotropic density variation distribution) measures the spread of the positive and negative regions related to CT. The CT index, i.e., the $t$ index difference between $D_{\mathrm{CT}}$ and the $H$ index, is another measure of the hole-electron separation (eqn (S15) and (S16) $\dagger$ ). The $D_{\mathrm{CT}}$ of Cz-DPVI, Cz-DMPVI, Cz-DEPVI and TPA-DEPVI was calculated to be $1.422,0.703,1.008$ and 0.352 , respectively (Fig. 7 and Table S11†). For both Cz-DEPVI and TPA-DEPVI, the non-zero $t$ is negative in all directions, where the overlap of holes and electrons is very severe (Fig. 7, Table S11 $\dagger$ ) and the eigenvalue is greater than 0.96 , which support the hybridization and is described in terms of dominant excitation pair as $94 \%$ transition.

This is further evidenced by the $\Delta r$ index (Tables S5 and S6 $\dagger$ ). The $\Delta r$ index (eqn $(\mathrm{S} 1) \dagger$ ) is the average hole $\left(\mathrm{h}^{+}\right)$-electron $\left(\mathrm{e}^{-}\right)$ distance $\left(d_{\mathrm{h}^{+}-\mathrm{e}^{-}}\right)$upon excitation, which indicates the nature of the excitation type, LE or CT. Specifically, valence excitation (LE) is related to short distances $\left(d_{\mathrm{h}^{+}-\mathrm{e}^{-}}\right)$, while larger distances $\left(d_{\mathrm{h}^{+}-}\right.$ $\left.\mathrm{e}^{-}\right)$are related to $\mathrm{CT}$ excitation. The triplet exciton is transformed into singlet excitons in TPA-DEPVI and Cz-DEPVI via the RISC process with a high energy excited state (hot CT channel) ${ }^{\mathbf{1 3 , 4 8}}$ which is beneficial for triplet exciton conversion in
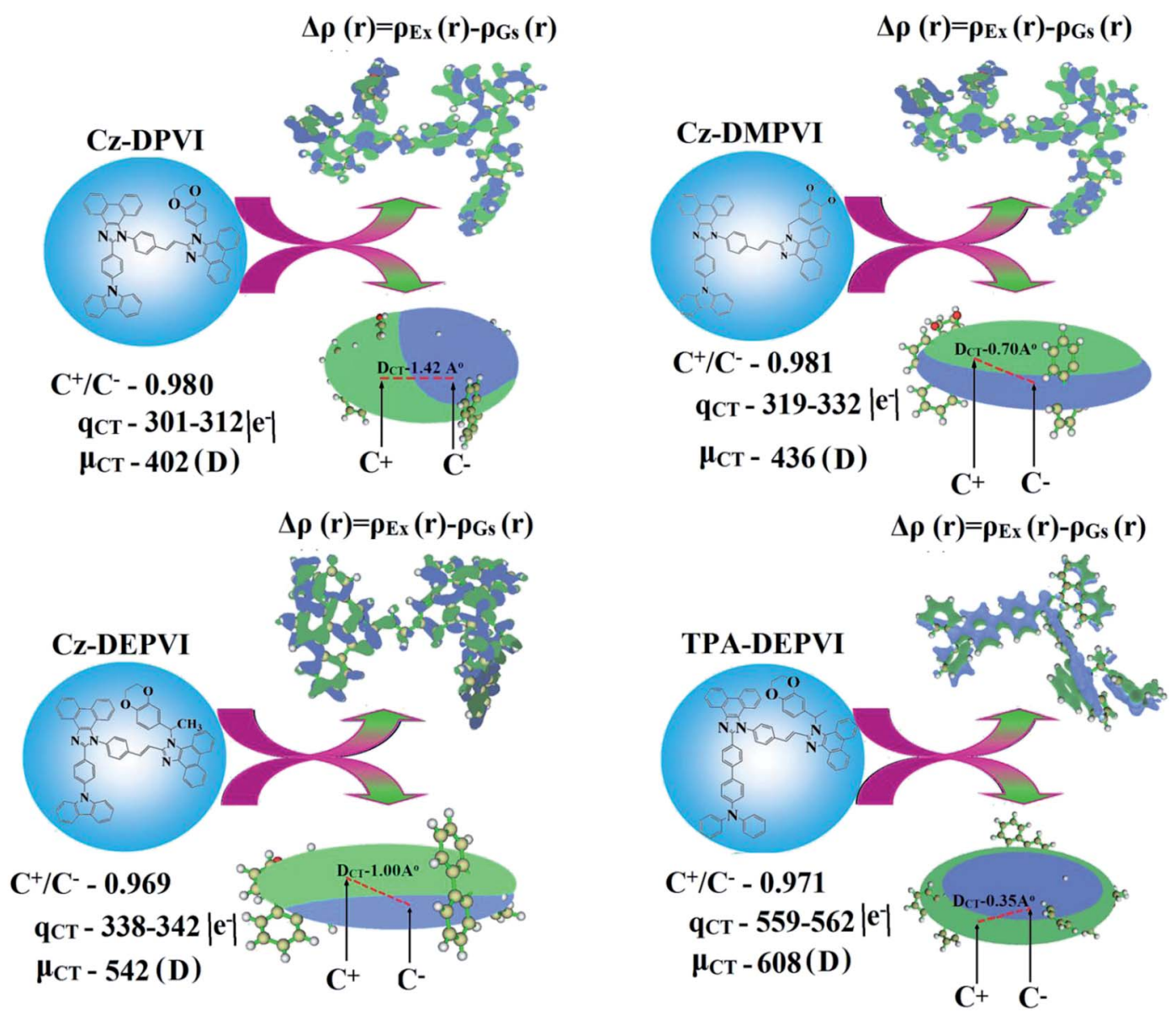

Fig. 7 Computed difference in total density for the ground and excited states $\left[\Delta \rho(r)=\rho_{\mathrm{Ex}}(r)-\rho_{\mathrm{Gs}}(r)\right.$; isosurface for Cz-DPVI, Cz-DMPVI, CzDEPVI, $\left(0.0000006\right.$ a.u.) and TPA-DEPVI $\left(0.15\right.$ a.u.)] and graphical representation of the $D_{C T}$ and centroid of charges $[C+(r) / C-(r) ;$ isosurface for Cz-DEPVI (0.29 a.u.) and TPA-DEPVI (0.1 a.u.)]. 

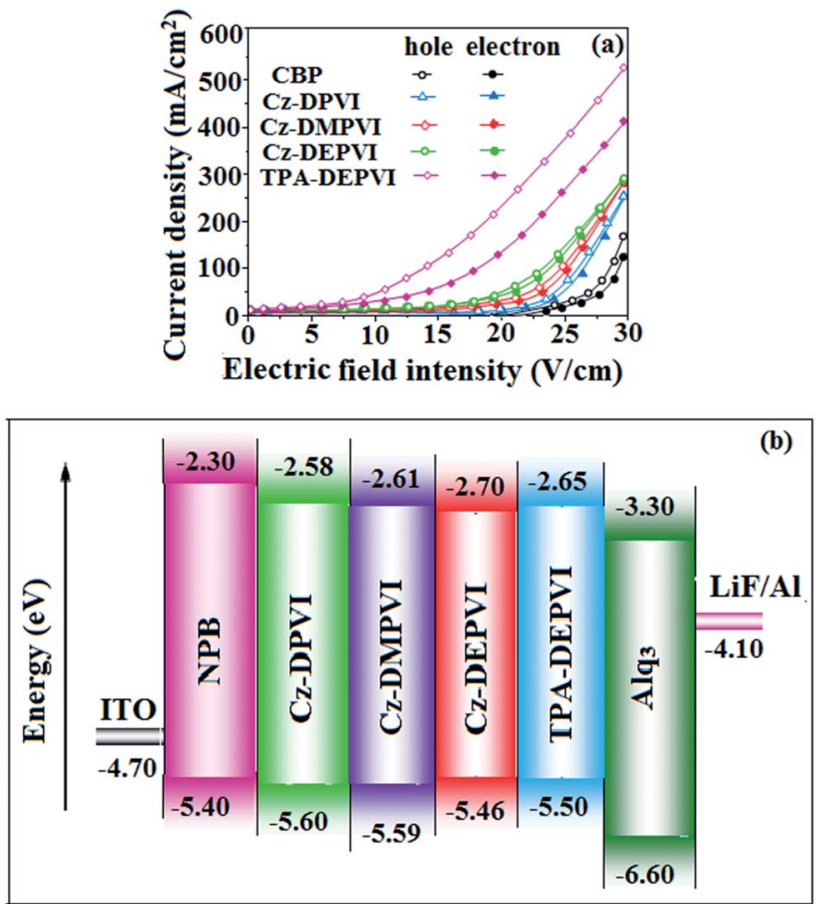

Fig. 8 (a) Hole-only and electron-only devices based on Cz-DPVI, Cz-DMPVI, Cz-DEPVI and TPA-DEPVI and (b) energy level diagram of the non-doped devices.

the electroluminescence process without any delayed fluorescence. The CT excitons are formed with a weak binding energy $\left(E_{\mathrm{b}}\right)$ on higher excited states. ${ }^{49} \mathrm{As}$ a result, the exciton utilization $\left(\eta_{\mathrm{S}}\right)$ can be harvested in TPA-DEPVI and Cz-DEPVI similarly to phosphorescent materials. The quasi-equivalent hybridized materials TPA-DEPVI and Cz-DEPVI exhibit excellent device performances due to the fine modulation in their excited states, where the enhanced LE component and hybridization between the LE and CT components result in high $\eta_{\mathrm{PL}}$ and high $\eta_{\mathrm{S}}$. Thus, the coexisting LE/CT composition in TPA-DEPVI and Cz-DEPVI harvested high $\eta_{\mathrm{PL}}$ and high $\eta_{\mathrm{S}}$ and enhanced the OLED performances (Table 1). These compounds exhibit blue emission with high quantum yields (solution/film), i.e., Cz-DPVI (0.76/0.83), Cz-DMPVI (0.80/0.89), Cz-DEPVI $(0.82 / 0.91)$ and TPA-DEPVI $(0.78 / 0.86)$ and high fluorescence efficiencies, which are essential for efficient blue OLEDs. The triplet energy $\left(E_{\mathrm{T}}\right)$ levels were estimated to be $2.34 \mathrm{eV}$ (Cz-DPVI), $2.40 \mathrm{eV}(\mathrm{Cz}-$ DMPVI), $2.49 \mathrm{eV}$ (Cz-DEPVI) and $2.46 \mathrm{eV}$ (TPA-DEPVI), which are sufficiently high for the excitation of green and red phosphorescent dopants. ${ }^{22}$ The $\Delta E_{\mathrm{ST}}$ of these hosts are relatively small, which is due to their spatially separated HOMO and LUMO levels. The small $\Delta E_{\mathrm{ST}}$ is advantageous for efficient energy transfer from the triplet excited state of the hosts to green and red phosphorescent emitters. ${ }^{27}$

To evaluate the carrier injection and transport properties of the materials, hole-only and electron-only devices were fabricated as follows: (a) ITO/HATCN(10 nm)/Cz-DPVI/Cz-DMPVI/CzDEPVI $(60 \mathrm{~nm}) / \mathrm{HATCN}(10 \mathrm{~nm}) / \mathrm{LiF}(1 \mathrm{~nm}) / \mathrm{Al}(100 \mathrm{~nm})($ holeonly device IV) and (b) ITO/TPBi $(10 \mathrm{~nm}) / \mathrm{Cz}-\mathrm{DPVI} / \mathrm{Cz}-\mathrm{DMPVI} /$ Cz-DEPVI $(60 \mathrm{~nm}) / \mathrm{TPBi} \quad(10 \mathrm{~nm}) / \mathrm{LiF} \quad(1 \mathrm{~nm}) / \mathrm{Al} \quad(100 \mathrm{~nm})$ (electron-only device V). Fig. 8 shows the current density versus voltage characteristics of the hole-only and electron-only devices. The electron current density of the Cz-DPVI-, CzDMPVI-, Cz-DEPVI- and TPA-DEPVI-based devices is higher than that for the CBP-based device. This reveals that these materials have better electron injection and transport properties than CBP. The difference in current density between the hole-only and electron-only devices based on Cz-DPVI, CzDMPVI, Cz-DEPVI and TPA-DEPVI is much smaller than that based on CBP at the same voltage, which suggests that these materials are potential bipolar materials capable of transporting electrons and holes in devices..$^{50-52}$

The observed intense blue emission and high $T_{\mathrm{g}}$ of Cz-DPVI, Cz-DMPVI, Cz-DEPVI and TPA-DEPVI support their suitability to serve as blue emitters in OLEDs. The device performances of the blue emitters were analysed by fabricating non-doped OLEDs with the configuration of ITO/NPB (1,4-bis(1naphthylphenylamino)-biphenyl) (50 nm)/Cz-DPVI/Cz-DMPVI/ Cz-DEPVI/TPA-DEPVI (30 nm)/BCP (2,9-dimethyl-4,7-diphenyl1,10-phenanthroline) $(15 \mathrm{~nm}) / \mathrm{Alq}_{3}$ (tris-(8-hydroxyquinoline) aluminum) $(50 \mathrm{~nm}) / \mathrm{LiF}(1 \mathrm{~nm}) / \mathrm{Al}(100 \mathrm{~nm})$ (Fig. 8). The device performances are displayed in Table 1. It is clear from Fig. 9 that the three new Cz-DPVI-, Cz-DMPVI-, Cz-DEPVI- and TPA-DEPVIbased devices exhibit high brightness at low voltage.

The EL spectra of the devices are similar to the PL spectra of Cz-DPVI, Cz-DMPVI, Cz-DEPVI and TPA-DEPVI in the film state (Fig. 2). The hole injection barrier between Cz-DEPVI and the hole transport layer is very small, and thus results in effective electron-hole radiative recombination in the emissive layer. The small injection barrier of Cz-DPVI ( $0.20 \mathrm{eV})$, Cz-DMPVI (0.19 eV), Cz-DEPVI ( $0.06 \mathrm{eV})$ and TPA-DEPVI $(0.10 \mathrm{eV})$ may account for their observed low turn-on voltages [Cz-DPVI $(3.4 \mathrm{~V}), \mathrm{Cz}^{-}$ DMPVI (3.2 V), Cz-DEPVI ( $2.8 \mathrm{~V})$ and TPA-DEPVI (2.9 V)]. The EQE of OLEDs can be calculated as follows: $\mathrm{EQE}=\eta_{\mathrm{out}} \times \eta_{\mathrm{rc}} \times$ $\eta_{\gamma} \times \Phi_{\mathrm{PL}},{ }^{53}$ where, $\eta_{\text {out }}$ is the light outcoupling efficiency (20\%), $\eta_{\mathrm{rc}}$ the product of the charge recombination efficiency (100\%), $\eta_{\gamma}$ the efficiency of radiative exciton production $(25 \%)$ and $\Phi_{\mathrm{PL}}$ the photoluminescence quantum yield of the emitters. The $\eta_{\mathrm{r}}$ for the Cz-DPVI-, Cz-DMPVI-, Cz-DEPVI- and TPA-DEPVI-based devices was calculated to be in the range of $20-27 \%$, which indicates $\gamma$ is less than $100 \%$ due to very small unbalanced carrier transportation. ${ }^{34}$ The maximum external quantum efficiency $\left(\eta_{\mathrm{ex}}\right)$ and current efficiency $\left(\eta_{\mathrm{c}}\right)$ of the Cz-DPVI-, CzDMPVI-, Cz-DEPVI- and TPA-DEPVI-based devices are $4.4 \%$, $4.6 \%, 4.9 \%$ and $4.7 \%$ and $4.9,5.4,6.0$ and $5.7 \mathrm{~cd} \mathrm{~A}^{-1}$, respectively. This result can be attributed to the more balanced charge-transporting properties within the emissive layer, which are achieved by the better charge injection provided by the hole transport layer. The Cz-DPVI-, Cz-DMPVI-, Cz-DEPVI- and TPADEPVI-based devices showed high power efficiencies $\left(\eta_{\mathrm{p}}\right)$ of $4.3(3.4 \mathrm{~V}), 5.0(3.2 \mathrm{~V}), 5.4(2.8 \mathrm{~V})$ and $5.2(2.9 \mathrm{~V}) \mathrm{lm} \mathrm{W}^{-1}$ with CIE coordinates of $(0.15,0.08),(0.15,0.08),(0.15,0.06)$ and $(0.15$, $0.07)$, respectively. The internal quantum efficiencies $\left(\eta_{\mathrm{IQE}}\right)$ of Cz-DPVI, Cz-DMPVI, Cz-DEPVI and TPA-DEPVI were calculated to be $22.0 \%, 23.0 \%, 24.5 \%$ and $23.5 \%$, and their exciton utilization efficiencies $\left(\eta_{\mathrm{S}}\right) 26.5 \%, 25.8 \%, 26.9 \%$ and $27.3 \%$, respectively. The high $\eta_{\mathrm{S}}$ of the Cz-DPVI-, Cz-DMPVI-, Cz-DEPVI- 

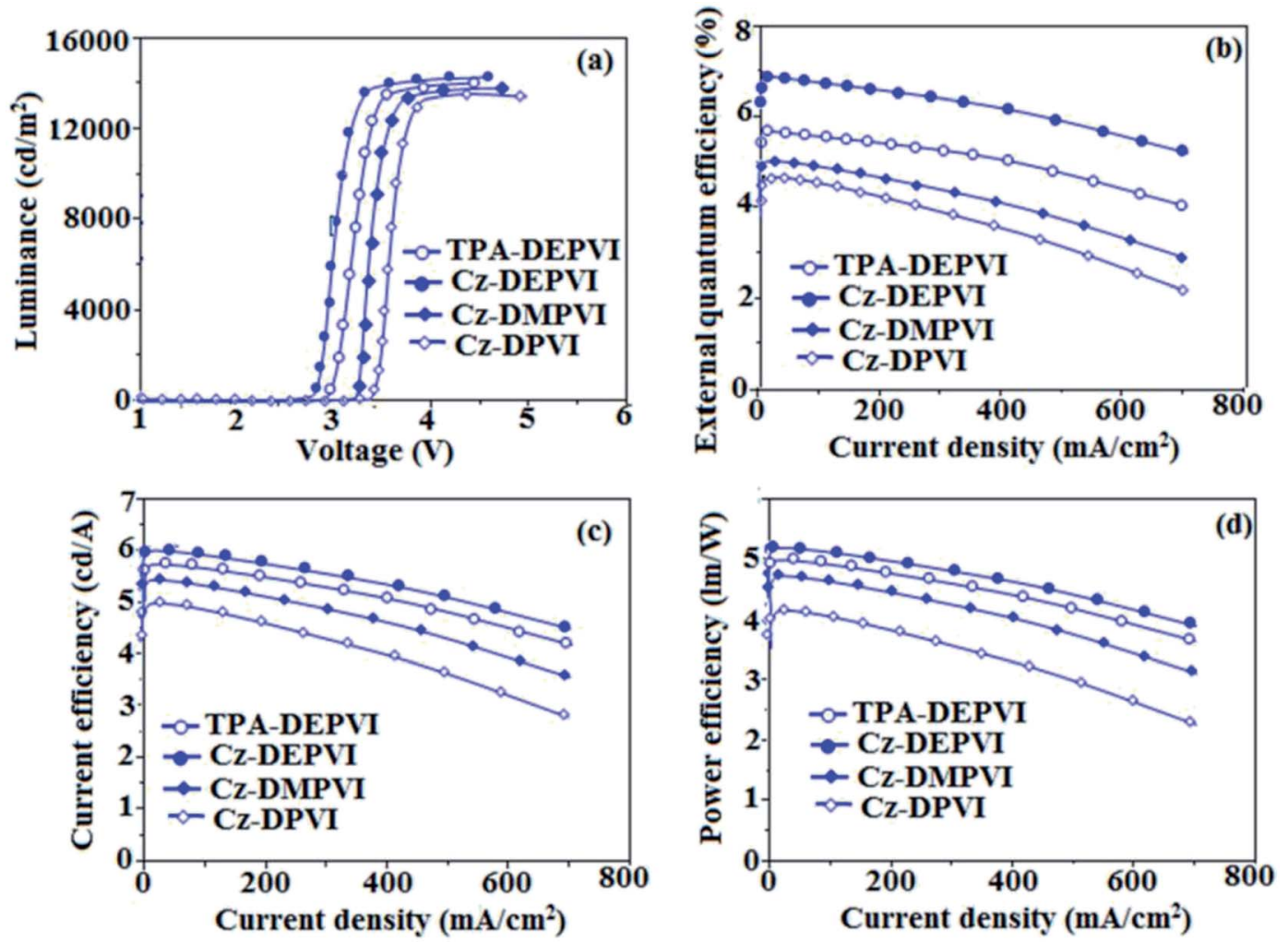

Fig. 9 Electroluminescence performances: (a) luminance versus voltage, (b) external quantum efficiency versus current density, (c) current efficiency versus current density and (d) power efficiency versus current density of Cz-DPVI, Cz-DMPVI, Cz-DEPVI and TPA-DEPVI.

and TPA-DEPVI-based devices is due to several different mechanisms, such as TADF, TTA and HLCT. Further, the luminance versus current density plot exhibits a linear relationship, and hence the high $\eta_{\mathrm{S}}$ cannot be attributed to the TTA process. The devices with Cz-DPVI, Cz-DMPVI, Cz-DEPVI and TPA-DEPVI showed the maximum luminance $(L)$ of 13 629, 13 841, 13955 and $13856 \mathrm{~cd} \mathrm{~m}^{-2}$, respectively. These experimental results demonstrate clearly that the additional triplet excitons were utilized in the OLED applications due to the HLCT character of Cz-DPVI, Cz-DMPVI, Cz-DEPVI and TPA-DEPVI, as shown in Scheme 1, and shows the accuracy of our molecular design strategy. The EL brightness exhibited a linear relationship with current density for these compounds, indicating that the contribution from triplet-triplet annihilation was insignificant. $^{54}$ The lifetime measurement revealed that this intercrossed excited state in different polar solvents should be a hybridized local and charge transfer state instead of a twospecies state through the simple addition of LE and CT. The mono-exponential lifetime [1.83 ns (Cz-DPVI), $1.92 \mathrm{~ns}\left(\mathrm{Cz}^{-}\right.$ DMPVI), $2.32 \mathrm{~ns}$ (Cz-DEPVI), and $2.10 \mathrm{~ns}$ (TPA-DEPVI)] demonstrates that the intercrossed LE and CT in the moderate polarity solvent formed one hybridized HLCT state, which supports the molecular design (Fig. 2). Since no delayed fluorescence was recorded from transient PL, the high EQEs did not seem to be in accordance with TADF. ${ }^{54}$ The emission wavelength of both Cz-DEPVI and TPA-DEPVI in film is close to that in ethyl ether, which supports the HLCT state formed in the
Cz-DEPVI and TPA-DEPVI films. The radiative transition rate $\left(k_{\mathrm{r}}\right)$ and the non-radiative transition rate $\left(k_{\mathrm{nr}}\right)$ of Cz-DEPVI and TPADEPVI were calculated using their lifetime and quantum yield values. The radiative $\left(k_{\mathrm{r}}=\phi / \tau\right) /$ non-radiative $\left(k_{\mathrm{nr}}=1 / \tau-(\phi / \tau)\right)$ $\left(k_{\mathrm{r}} / k_{\mathrm{nr}} 10^{8} \mathrm{~s}^{-1}\right)$ rate constants of Cz-DPVI (4.15/1.31), Cz-DMPVI (4.17/1.03), Cz-DEPVI (3.53/0.78) and TPA-DEPVI (3.71/1.05) (Table 1) reveal that $k_{\mathrm{r}}>k_{\mathrm{nr}}$. Compared with TPA-DEPVI, the $k_{\mathrm{r}}$ of Cz-DEPVI increased and the $k_{\mathrm{nr}}$ of Cz-DEPVI decreased. This result is also in good agreement with the aim of our molecular design.

The current and power efficiencies of the devices based on Cz-DEPVI $\left(6.0 \mathrm{~cd} \mathrm{~A}^{-1}\right.$ and $\left.5.4 \mathrm{~lm} \mathrm{~W}^{-1}\right)$ and TPA-DEPVI $(5.7 \mathrm{~cd}$ $\mathrm{A}^{-1}$ and $5.2 \mathrm{~lm} \mathrm{~W}^{-1}$ ) are higher than that of the devices based on TPA-PA (1.16 $\mathrm{cd} \mathrm{A}^{-1}$ and $\left.0.65 \mathrm{~lm} \mathrm{~W}^{-1}\right)$, TPA-NzP $\left(1.00 \mathrm{~cd} \mathrm{~A}^{-1}\right.$ and $\left.0.77 \mathrm{~lm} \mathrm{~W}^{-1}\right)^{55}$ and $m$ TPA-PPI $\left(0.84 \mathrm{~cd} \mathrm{~A}^{-1}\right.$ and $0.48 \mathrm{~lm}$ $\left.\mathrm{W}^{-1}\right)$, respectively. Also the external quantum yield of Cz-DEPVI $(82 \%)$ and Cz-DEPVI (78\%) is higher than that of (i) Cz-BzP (69.7\%) and TPA-BzP $(49.2 \%)^{56}$ (ii) CBI (21\%) and MCB $(24 \%)$ and (iii) PPI-pCNCz (54\%). Wang et al. ${ }^{57}$ reported that the thickness of the LBPPI emissive layer alters the current efficiency (50 nm: $0.01 \mathrm{~cd} \mathrm{~A}^{-1}, 40 \mathrm{~nm}: 0.13 \mathrm{~cd} \mathrm{~A}^{-1}, 30 \mathrm{~nm}: 0.40 \mathrm{~cd}$ $\mathrm{A}^{-1}$ and $20 \mathrm{~nm}: 0.68 \mathrm{~cd} \mathrm{~A}^{-1}$ ). The current efficiency obtained in the present study with a thickness of $30 \mathrm{~nm}$ for Cz-DEPVI $(6.0 \mathrm{~cd}$ $\left.\mathrm{A}^{-1}\right)$ and $30 \mathrm{~nm}$ for TPA-DEPVI $\left(5.7 \mathrm{~cd} \mathrm{~A}^{-1}\right)$ is higher than that previously reported..$^{5-57}$ Hence, it is possible to improve the efficiency of these materials through modification of the thickness of the emissive layer. Accordingly, efforts will be made 

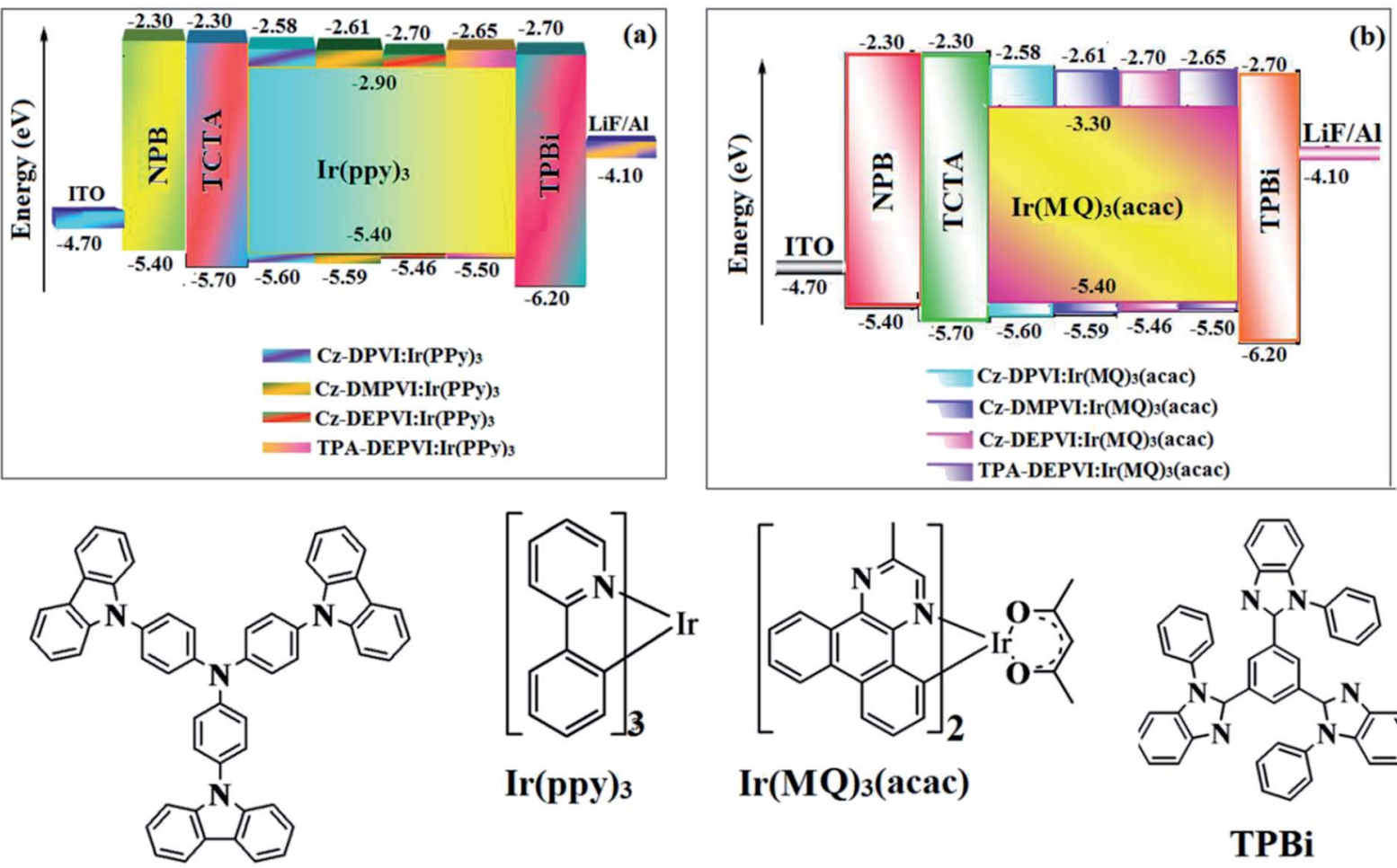<smiles>c1ccc2c(c1)-c1ccccc1C21C[In]1</smiles>

$\operatorname{Ir}(\text { ppy })_{3}$

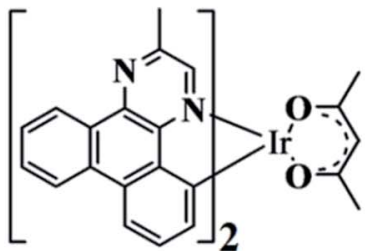

$\operatorname{Ir}(\mathrm{MQ})_{3}(\mathbf{a c a c})$

TCTA

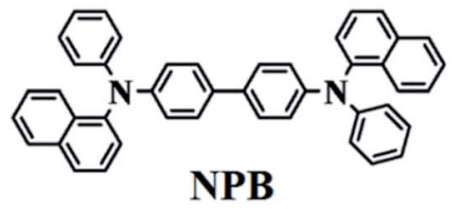

Fig. 10 Energy level diagrams of the green (a) and red (b) devices with the molecular structures of the functional materials used in the devices.

to modify the thickness of the emissive layer to enhance the efficiency and to increase the radiative rate in our future studies. The weak donor carbazole-substituted phenanthrimidazole exhibits a current efficiency of $0.88 \mathrm{~cd} \mathrm{~A}^{-1}$ and power efficiency of $0.30 \mathrm{~lm} \mathrm{~W}^{-1}$ and Gao et al. reported a carbazole-substituted compound with a current efficiency of $0.65 \mathrm{~cd} \mathrm{~A}^{-1}$ and power efficiency of $0.48 \mathrm{~lm} \mathrm{~W}^{-1} .^{57}$ These carbazole-substituted compounds exhibited lower efficiencies and power efficiencies than that obtained in our studies with Cz-DPVI (4.9 $\mathrm{cd} \mathrm{A}^{-1}$ and $4.3 \mathrm{~lm} \mathrm{~W}^{-1}$ ), Cz-DMPVI (5.4 $\mathrm{cd} \mathrm{A}^{-1}$ and $\left.5.0 \mathrm{~lm} \mathrm{~W}^{-1}\right)$ and Cz-DEPVI (6.0 $\mathrm{cd} \mathrm{A}^{-1}$ and $\left.5.4 \mathrm{~lm} \mathrm{~W}^{-1}\right)$, respectively. The device efficiency indicates that $\mathrm{Cz}-\mathrm{DPVI}, \mathrm{Cz}-$ DMPVI, Cz-DEPVI and TPA-DEPVI are excellent fluorescent OLED materials.

Cz-DPVI, Cz-DMPVI, Cz-DEPVI and TPA-DEPVI were also employed as host materials for green and red phosphorescent dopants. The fabricated green and red devices had the configuration of ITO/NPB (40 nm)/TCTA $(5 \mathrm{~nm}) / \mathrm{Cz}-\mathrm{DPVI}(30 \mathrm{~nm})$ : 5 wt $\% \operatorname{Ir}(\mathrm{ppy})_{3} / \mathrm{Cz}$-DMPVI $(30 \mathrm{~nm}): 5 \mathrm{wt} \% \operatorname{Ir}(\mathrm{ppy})_{3} / \mathrm{Cz}$-DEPVI (30 $\mathrm{nm}): 5 \mathrm{wt} \% \operatorname{Ir}(\mathrm{ppy})_{3} / \mathrm{TPA}-\mathrm{DEPVI}(30 \mathrm{~nm}): 5 \mathrm{wt} \% \operatorname{Ir}(\mathrm{ppy})_{3}$ TPBI $(50$ $\mathrm{nm}) / \mathrm{LiF}(1 \mathrm{~nm}) / \mathrm{Al}(100 \mathrm{~nm})]:$ ITO/NPB $(40 \mathrm{~nm}) / \mathrm{TCTA}(5 \mathrm{~nm}) / \mathrm{Cz}-$ DPVI (30 nm): 8 wt $\% \operatorname{Ir}(\mathrm{MQ})_{2}($ acac)/Cz-DMPVI $(30 \mathrm{~nm}): 8 \mathrm{wt} \%$ $\operatorname{Ir}(\mathrm{MQ})_{2}(\mathrm{acac}) / \mathrm{Cz}-\mathrm{DEPVI} \quad(30 \mathrm{~nm}): 8 \mathrm{wt} \% \quad \operatorname{Ir}(\mathrm{MQ})_{2}(\mathrm{acac}) / \mathrm{TPA}-$ DEPVI (30 nm): $8 \mathrm{wt} \% \operatorname{Ir}(\mathrm{MQ})_{2}(\mathrm{acac}) / \mathrm{TPBI}(50 \mathrm{~nm}) / \mathrm{LiF}(1 \mathrm{~nm}) /$ Al (100 nm)], respectively (Fig. 10), and $\operatorname{Ir}(\mathrm{ppy})_{3}$-fac-tris(2phenylpyridine) iridium(III) and $\operatorname{Ir}(\mathrm{MQ})_{2}$ (acac)-bis(2- methyldibenzo- $[f, h]$ quinoxaline) acetylacetonate iridium(III) were used as emissive layers for the green and red devices, respectively.

The device performances are shown in Fig. 11. The EL spectra are similar to the PL spectra of the doped thin films (Fig. 2). The green device based on Cz-DEPVI (30 nm): $5 \mathrm{wt} \%$ $\operatorname{Ir}(\mathrm{ppy})_{3}$ exhibited the maximum luminance of $8891 \mathrm{~cd} \mathrm{~m}^{-2}$, and maximum current and power efficiencies of $27.9 \mathrm{~cd} \mathrm{~A}^{-1}$ and $33.4 \mathrm{~lm} \mathrm{~W}^{-1}$, respectively at $2.7 \mathrm{~V}$ (Table 3 ). The maximum external quantum efficiencies of the devices based on $\mathrm{Cz}$ DPVI:Ir(ppy) ${ }_{3}, \mathrm{Cz}-\mathrm{DMPVI}: \operatorname{Ir}(\mathrm{ppy})_{3}, \mathrm{Cz}$-DEPVI:Ir(ppy) ${ }_{3}$ and TPADEPVI:Ir(ppy $)_{3}$ are $18.2 \%, 18.9 \%, 19.3 \%$ and $19.0 \%$, respectively. Similar to the green devices, red device based on $\mathrm{Cz}$ DEPVI:Ir(MQ) $)_{2}$ (acac) exhibited the maximum luminance of $40565 \mathrm{~cd} \mathrm{~m}^{-2}$ and excellent EL efficiencies $\left(\eta_{\mathrm{ex}}: 19.9 \%, \eta_{\mathrm{c}}: 26.0\right.$ $\mathrm{cd} \mathrm{A}^{-1}$, and $\left.\eta_{\mathrm{p}}: 30.4 \mathrm{~lm} \mathrm{~W}^{-1}\right)$ with CIE coordinates of $(0.64,0.37)$ among the red devices, including Cz-DPVI:Ir(MQ) $)_{2}(\mathrm{acac})(L$ : $17215 \mathrm{~cd} \mathrm{~m}^{-2}$, $\eta_{\mathrm{ex}}: 17.6 \%, \eta_{\mathrm{c}}: 21.1 \mathrm{~cd} \mathrm{~A}^{-1}$, and $\eta_{\mathrm{p}}: 25.4 \mathrm{~lm} \mathrm{~W}^{-1}$ with CIE coordinates of $(0.64,0.37))$, Cz-DMPVI:Ir(MQ) $)_{2}$ (acac) $\left(L: 20628 \mathrm{~cd} \mathrm{~m}^{-2}, \eta_{\mathrm{ex}}: 19.0 \%, \eta_{\mathrm{c}}: 24.9 \mathrm{~cd} \mathrm{~A}^{-1}\right.$, and $\eta_{\mathrm{p}}: 26.8 \mathrm{~lm}$ $\mathrm{W}^{-1}$ with CIE coordinates of $\left.(0.64,0.37)\right)$ and TPADEPVI:Ir(MQ) $)_{2}$ (acac) ( $L: 39865 ; \mathrm{cd} \mathrm{m}^{-2}, \eta_{\mathrm{ex}}: 19.3 \%, \eta_{\mathrm{c}}: 25.4 \mathrm{~cd}$ $\mathrm{A}^{-1}, \eta_{\mathrm{p}}: 26.2 \mathrm{~lm} \mathrm{~W}^{-1}$ with CIE coordinates of $\left.(0.64,0.37)\right)$. The above experimental results demonstrate that $\mathrm{Cz}-\mathrm{DPVI}, \mathrm{Cz}-$ DMPVI, Cz-DEPVI and TPA-DEPVI are universal host materials for green and red phosphorescent emitters (Table 3). 

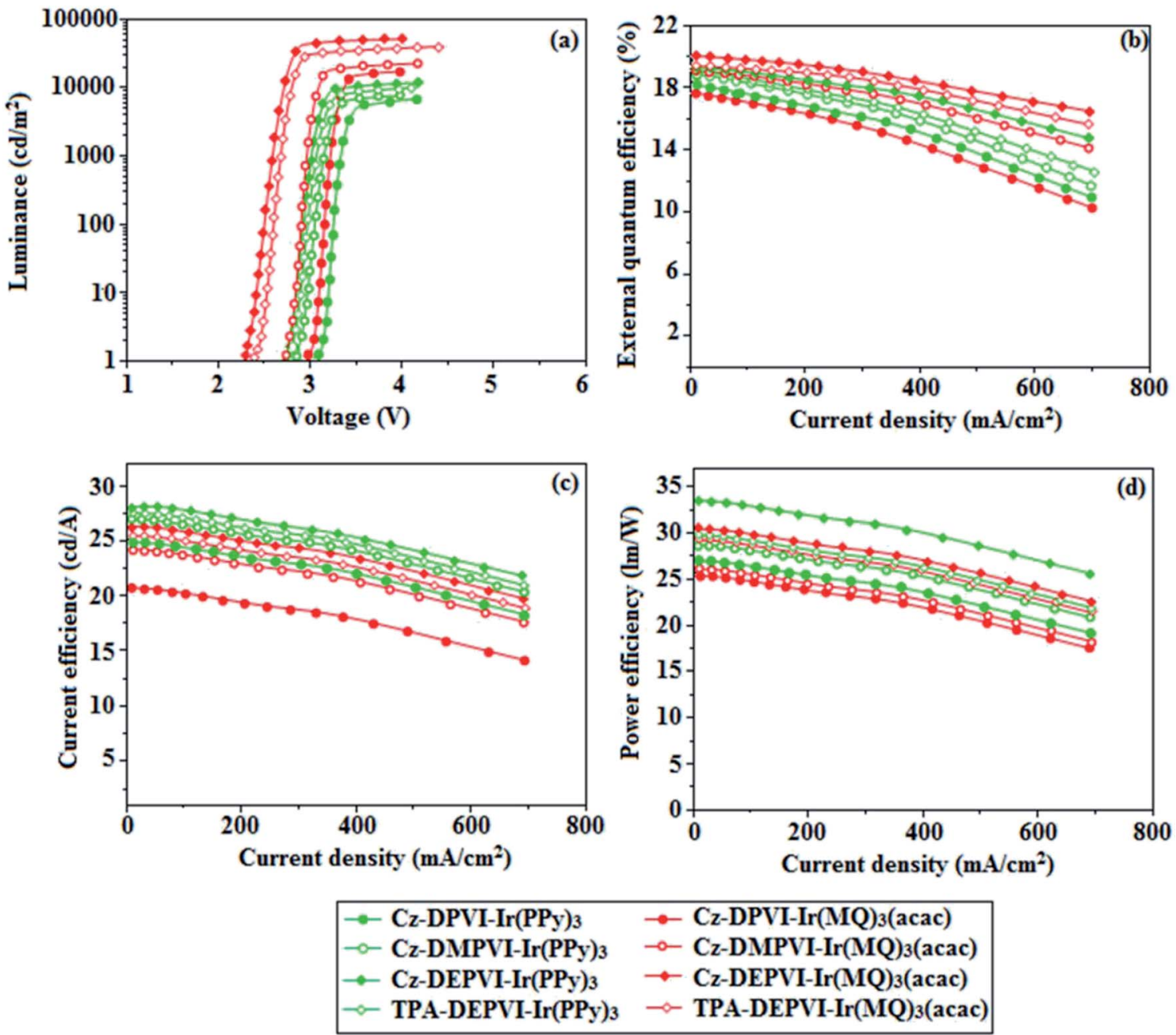

Fig. 11 Device efficiencies: (a) Luminance versus voltage, (b) external quantum efficiency versus current density, (c) current efficiency versus current density and (d) power efficiency versus current density of the green devices based on Cz-DPVI:Ir(PPy) ${ }_{3}, \mathrm{Cz}-\mathrm{DMPVI}: \mathrm{Ir}(\mathrm{PPy})_{3}, \mathrm{Cz}-$ DEPVI:Ir(PPy) ${ }_{3}$ and TPA-DEPVI:Ir(PPy) ${ }_{3}$ and red devices based on Cz-DPVI:Ir(MQ) $)_{3}(\mathrm{acac}), \mathrm{Cz}-\mathrm{DMPVI}: \operatorname{Ir}(\mathrm{MQ})_{3}(\mathrm{acac}), \mathrm{Cz}-\mathrm{DEPVI}: \operatorname{Ir}(\mathrm{MQ})_{3}(\mathrm{acac})$ and TPA-DEPVI:Ir(MQ) ${ }_{3}(\mathrm{acac})$.

\section{Conclusion}

We reported efficient new deep blue-emitting materials $\mathrm{Cz}$ DPVI, Cz-DMPVI, Cz-DEPVI and TPA-DEPVI with the D-A geometry, which exhibit dual charge transport properties and show excellent thermal properties with high glass-transition temperature. The photophysical properties, film morphology, and electrochemical and electroluminescent properties of $\mathrm{Cz}$ DEPVI and TPA-DEPVI can be tuned by chemical modification from $\mathrm{Cz}$-DEPVI to TPA-DEPVI by changing the strong donor TPA moiety with the weak donor $\mathrm{Cz}$ moiety. The deep blue emission with carrier transport abilities of Cz-DPVI, Cz-DMPVI, Cz-DEPVI and TPA-DEPVI reveal that the non-doped devices with these compounds exhibit maximum external quantum efficiencies, and current and power efficiencies, i.e., Cz-DPVI (4.4\%, $4.9 \mathrm{~cd}$ $\mathrm{A}^{-1}$ and $4.3 \mathrm{~lm} \mathrm{~W}^{-1}$ ), Cz-DMPVI (4.6\%, $5.4 \mathrm{~cd} \mathrm{~A}^{-1}$ and $5.0 \mathrm{~lm}$ $\mathrm{W}^{-1}$ ), Cz-DEPVI $\left(4.9 \%, 6.0 \mathrm{~cd} \mathrm{~A}^{-1}\right.$ and $\left.5.4 \mathrm{~lm} \mathrm{~W}^{-1}\right)$ and TPADEPVI $\left(4.7 \%, 5.7 \mathrm{~cd} \mathrm{~A}^{-1}\right.$ and $\left.5.2 \mathrm{~lm} \mathrm{~W}^{-1}\right)$, respectively. These materials exhibit blue emission with CIE coordinates of $(0.15$, 0.08 ) for Cz-DPVI, $(0.15,0.08)$ for Cz-DMPVI, $(0.15,0.06)$ for $\mathrm{Cz}$ -
DEPVI and $(0.15,0.07)$ for TPA-DEPVI at a low driving voltage. These blue emissive materials with good carrier transport properties were also employed as hosts for green and red phosphorescent devices. The maximum external quantum efficiencies of the devices based on Cz-DPVI:Ir(ppy) $)_{3}, \mathrm{Cz}-$ DMPVI:Ir(ppy $)_{3}, \quad$ Cz-DEPVI:Ir(ppy $)_{3}$ and TPA-DEPVI:Ir(ppy) ${ }_{3}$ were $18.2 \%, 18.9 \%, 19.3 \%$ and $19.0 \%$, respectively. Similarly to the green devices, the red device based on Cz-DEPVI:Ir(MQ) ${ }_{2}(-$ acac) exhibited the maximum luminance of $40565 \mathrm{~cd} \mathrm{~m}^{-2}$ and excellent EL efficiencies $\left(\eta_{\mathrm{ex}}: 19.9 \%, \eta_{\mathrm{c}}: 26.0 \mathrm{~cd} \mathrm{~A}^{-1}, \eta_{\mathrm{p}}: 30.4 \mathrm{~lm}\right.$ $\left.\mathrm{W}^{-1}\right)$ with CIE coordinates of $(0.64,0.37)$. The present findings demonstrate a new route to harvest high-performance full-color OLEDs by utilizing bipolar luminescent materials possessing the D-A molecular structure. Also, the crucial role of host materials with high triplet energy and the small $\Delta_{\mathrm{ST}}$ strategy can be used for the development of efficient green and red OLEDs.

\section{Conflicts of interest}

There are no conflicts to declare. 


\section{Acknowledgements}

One of the authors Dr J. Jayabharathi thanks Department of Science and Technology (EMR/2014/000094), Defence Research and Development Organization (213/MAT/10-11), Council of Scientific and Industrial Research [No. 01/(2707)/13EMR-II], University Grant Commission (36-21/2008) and Nano Mission (SR/NM/NS-1001/2016) for financial support. Authors would like to thank Mr P. Justin Jesuraj for scientific discussions related to device performances.

\section{References}

1 (a) Y. Sun, N. C. Giebink, H. Kanno, B. Ma, M. E. Thompson and S. R. Forrest, Nature, 2006, 440, 908-912; (b) S. Reineke, F. Lindner, G. Schwartz, N. Seidler, K. Walzer, B. Lussem and K. Leo, Nature, 2009, 459, 234-238; (c) K. Kreger, M. Bate, C. Neuber, H. W. Schmidt and P. Strohriegl, Adv. Funct. Mater., 2007, 17, 3456-3461; (d) C. J. Zheng, J. Wang, J. Ye, M. F. Lo, X. K. Liu, M. K. Fung, X. H. Zhang and C. S. Lee, Adv. Mater., 2013, 25, 2205-2211.

2 (a) J. Ding, J. Gao, Y. Cheng, Z. Xie, L. Wang, D. Ma, X. Jing and F. Wang, Adv. Funct. Mater., 2006, 16, 575-581; (b) H. Sasabe, J. Takamatsu, T. Motoyama, S. Watanabe, G. Wagenblast, N. Langer, O. Molt, E. Fuchs, C. Lennartz and J. Kido, Adv. Mater., 2010, 22, 5003-5007.

3 (a) J. Huang, N. Sun, Y. Dong, R. Tang, P. Lu, P. Cai, Q. Li, D. Ma, J. Qin and Z. Li, Adv. Funct. Mater., 2013, 23, 23292337; (b) C. J. Tonzola, A. P. Kulkarni, A. P. Gifford, W. Kaminsky and S. A. Jenekhe, Adv. Funct. Mater., 2007, 17, 863-874; (c) C. H. Chien, C. K. Chen, F. M. Hsu, C. F. Shu, P. T. Chou and C. H. Lai, Adv. Funct. Mater., 2009, 19, 560-566.

4 (a) K. Guo, J. Zhang, T. Xu, X. Gao and B. Wei, J. Disp. Technol., 2014, 10, 642-646; (b) M. Liu, X. L. Li, D. C. Chen, Z. Z. Xie, X. Y. Cai, G. Z. Xie, K. K. Liu, J. X. Tang, S. J. Su and Y. Cao, Adv. Funct. Mater., 2015, 25, 5190-5198.

5 (a) Y. Cao, I. D. Parker, G. Yu, C. Zhang and A. J. Heeger, Nature, 1999, 397, 414-417; (b) H. Uoyama, K. Goushi, K. Shizu, H. Nomura and C. Adachi, Nature, 2012, 492, 234-238; (c) L. Yao, S. Zhang, R. Wang, W. Li, F. Shen, B. Yang and Y. Ma, Angew. Chem., Int. Ed., 2014, 53, 21192123; (d) X. H. Ouyang, X. L. Li, L. Ai, D. B. Mi, Z. Y. Ge and S. J. Su, ACS Appl. Mater. Interfaces, 2015, 7, 7869-7877; (e) Z. Gao, G. Cheng, F. Shen, S. Zhang, Y. Zhang, P. Lu and Y. Ma, Laser Photonics Rev., 2014, 8, L6-L10.

6 (a) A. D. Gorse and M. Pesquer, J. Phys. Chem., 1995, 99, 4039-4049; (b) W. Y. Hung, G. C. Fang, Y. C. Chang, T. Y. Kuo, P. T. Chou, S. W. Lin and K. T. Wong, ACS Appl. Mater. Interfaces, 2013, 5, 6826-6831.

7 (a) Y. Tao, K. Yuan, T. Chen, P. Xu, H. Li, R. Chen, C. Zheng, L. Zhang and W. Huang, Adv. Mater., 2014, 26, 7931-7958; (b) W. Chen, Y. Yuan, Z. Zhu, Z. Jiang, L. Liao and C. Lee, $A d v$. Opt. Mater., 2018, 6, 1700855-1700863; (c) A. Li, Z. Ma, J. Wu, P. Li, H. Wang, Y. Geng, S. Xu, B. Yang, H. Zhang, H. Cui and W. Xu, Adv. Opt. Mater., 2018, 6, 17006471700655; (d) L. Cui, H. Nomura, Y. Geng, J. Kim,
H. Nakanotani and C. Adachi, Angew. Chem., 2017, 56, 1571-1575; (e) T. A. Lin, T. Chatterjee, W. L. Tsai, W. K. Lee, M. J. Wu, M. Jiao, K. C. Pan, C. L. Yi, C. L. Chung, K. T. Wong and C. C. Wu, Adv. Mater., 2016, 28, 6976-6983; (f) K. C. Pan, S. W. Li, Y. Y. Ho, Y. J. Shiu, W. L. Tsai, M. Jiao, W. K. Lee, C. C. Wu, C. L. Chung, T. Chatterjee, Y. S. Li, K. T. Wong, H. C. Hu, C. C. Chen and M. T. Lee, Adv. Funct. Mater., 2016, 26, 7560-7571.

8 J. Li, T. Nakagawa, J. MacDonald, Q. Zhang, H. Nomura, H. Miyazaki and C. Adachi, Adv. Mater., 2013, 25, 3319-3323.

9 W. J. Li, D. D. Liu, F. Z. Shen, D. G. Ma, Z. M. Wang, T. Fei, B. Yang and Y. G. Ma, Adv. Funct. Mater., 2012, 22, 27972803.

10 W. J. Li, Y. Y. Pan, R. Xiao, Q. M. Peng, S. T. Zhang, D. G. Ma, F. Li, F. Z. Shen, Y. H. Wang, B. Yang and Y. G. Ma, Adv. Funct. Mater., 2014, 24, 1609-1614.

11 S. Tang, W. J. Li, F. Z. Shen, D. D. Liu, B. Yang and Y. G. Ma, J. Mater. Chem., 2012, 22, 4401-4408.

12 S. T. Zhang, W. J. Li, L. Yao, Y. Y. Pan, B. Yang and Y. G. Ma, Chem. Commun., 2013, 49, 11302-11304.

13 L. Yao, S. T. Zhang, R. Wang, W. J. Li, F. Z. Shen, B. Yang and Y. G. Ma, Angew. Chem., Int. Ed., 2014, 126, 2151-2155.

14 Y. Y. Pan, W. J. Li, S. T. Zhang, L. Yao, C. Gu, H. Xu, B. Yang and Y. G. Ma, Adv. Opt. Mater., 2014, 2, 510-515.

15 W. J. Li, Y. Y. Pan, L. Yao, H. C. Liu, S. T. Zhang, C. Wang, F. Z. Shen, B. Yang and Y. G. Ma, Adv. Opt. Mater., 2014, 2, 892-910.

16 D. Chaudhuri, E. Sigmund, A. Meyer, L. Rcck, P. Klemm, S. Lautenschlager, A. Schmid, S. R. Yost, T. Van, S. Bange, S. Hcger and J. M. Lupton, Angew. Chem., 2013, 125, 13691-13694.

17 J. R. Sheats, H. Antoniadis, M. H. W. Leonard, J. Miller, R. Moon, D. Roitman and A. Stocking, Science, 1996, 273, 884-888.

18 S. Zhang, L. Yao, Q. Peng, W. Li, Y. Pan, R. Xiao, Y. Gao, C. Gu, Z. Wang, P. Lu, F. Li, S. Su, B. Yang and Y. Ma, Adv. Funct. Mater., 2015, 25, 1755-1762.

19 M. A. Baldo, S. Lamansky, P. E. Burrows, M. E. Thompson and S. R. Forrest, Appl. Phys. Lett., 1999, 60, 14422-14428.

20 T. Forster, 10th Spiers Memorial Lecture, Discuss. Faraday Soc., 1959, 27, 7-17.

21 (a) K. Wang, F. C. Zhao, C. G. Wang, S. Y. Chen, D. Chen, H. Y. Zhang, Y. Liu, D. G. Ma and Y. Wang, Adv. Funct. Mater., 2013, 23, 2672-2680; (b) K. Wang, S. P. Wang, J. B. Wei, S. Y. Chen, D. Liu, Y. Liu and Y. Wang, J. Mater. Chem. C, 2014, 2, 6817-6826.

22 (a) M. J. Frisch, G. W. Trucks, H. B. Schlegel, G. E. Scuseria, M. A. Robb, J. R. Cheeseman, J. A. Montgomery, T. Vreven, K. N. Kudin, J. C. Burant, J. M. Millam, S. S. Iyengar, J. Tomasi, V. Barone, B. Mennucci, M. Cossi, G. Scalmani, N. Rega, G. A. Petersson, H. Nakatsuji, M. Hada, M. Ehara, K. Toyota, R. Fukuda, J. Hasegawa, M. Ishida, T. Nakajima, Y. Honda, O. Kitao, H. Nakai, M. Klene, X. Li, J. E. Knox, H. P. Hratchian, J. B. Cross, V. Bakken, C. Adamo, J. Jaramillo, R. Gomperts, R. E. Stratmann, O. Yazyev, A. J. Austin, R. Cammi, C. Pomelli, J. W. Ochterski, P. Y. Ayala, K. Morokuma, G. A. Voth, P. Salvador, 
J. J. Dannenberg, V. G. Zakrzewski, S. Dapprich, A. D. Daniels, M. C. Strain, O. Farkas, D. K. Malick, A. D. Rabuck, K. Raghavachari, J. B. Foresman, J. V. Ortiz, Q. Cui, A. G. Baboul, S. Clifford, J. Cioslowski, B. B. Stefanov, G. Liu, A. Liashenko, P. Piskorz, I. Komaromi, R. L. Martin, D. J. Fox, T. Keith, M. A. A. Laham, C. Y. Peng, A. Nanayakkara, M. Challacombe, P. M. W. Gill, B. Johnson, W. Chen, M. W. Wong, C. Gonzalez and J. A. Pople, Gaussian 09 (Revision A.02), Gaussian, Inc., Wallingford, CT. 2009; (b) T. Lu and F. Chen, J. Comput. Chem., 2012, 33, 580-592.

23 M. S. Tsai, Y. C. Hsu, J. T. Lin, H. C. Chen and C. P. Hsu, J. Phys. Chem. C, 2007, 111, 18785-18793.

24 Z. Gao, Y. Liu, Z. Wang, F. Shen, H. Liu, G. Sun, L. Yao, Y. Lv, P. Lu and Y. Ma, Chem.-Eur. J., 2013, 19, 2602-2605.

25 C. Li, S. Wang, W. Chen, J. Wei, G. Yang, K. Ye, Y. Liu and Y. Wang, Chem. Commun., 2015, 51, 10632-10635.

26 W. Sun, N. Zhou, Y. Xiao, S. Wang and X. Li, Chem.-Asian J., 2017, 12, 3069-3076.

27 Y. Tao, Q. Wang, C. Yang, C. Zhong, K. Zhang, J. Qin and D. Ma, Adv. Funct. Mater., 2010, 20, 304-311.

28 C. Fan, Y. H. Chen, Z. Q. Jiang, C. L. Yang, C. Zhong, J. G. Qin and D. G. Ma, J. Mater. Chem., 2010, 20, 3232-3237.

29 Z. Huang, B. Wang, Q. Zhang, S. Xiang, X. Lv, L. Ma, B. Yang, Y. Gao and L. Wang, Dyes Pigm., 2017, 140, 328-336.

30 P. Y. Chou, H. H. Chou, Y. H. Chen, et al., Chem. Commun., 2014, 50, 6869-6871.

31 J. Jayabharathi, P. Ramanathan and V. Thanikachalam, New J. Chem., 2015, 39, 142-154.

32 T. Shan, Z. Gao, X. Tong, X. He, Y. Gao, J. Li, X. Sun, Y. Liu, H. Liu, B. Yang, P. Lu and Y. Ma, Dyes Pigm., 2017, 142, 189197.

33 J. Herbich and A. Kapturkiewiez, J. Am. Chem. Soc., 1998, 120, 1014-1029.

34 W. C. Chen, Y. Yuan, G. F. Wu, H. X. Wei, J. Ye, M. Chen, F. Lu, Q. X. Tong, F. Wong and C. Lee, Org. Electron., 2015, 17, 159-166.

35 (a) L. Duan, J. Qiao, Y. D. Sun and Y. Qiu, Adv. Mater., 2011, 23, 1137-1144; (b) D. D. Zhang, L. Duan, Y. L. Li, H. Y. Li, Z. Y. Bin, D. Q. Zhang, J. Qiao, G. D. Dong, L. D. Wang and Y. Qiu, Adv. Funct. Mater., 2014, 24, 3551-3561.

36 Y. Yuan, J. X. Chen, F. Lu, Q. X. Tong, Q. D. Yang, H. W. Mo, T. W. Ng, F. L. Wong, Z. Q. Guo, J. Ye, Z. Chen, X. H. Zhang and C. S. Lee, Chem. Mater., 2013, 25, 4957-4965.

37 T. H. Huang, J. T. Lin, L. Y. Chen, Y. T. Lin and C. C Wu, Adv. Mater., 2006, 18, 602-606.

38 K. C. Wu, P. J. Ku, C. S. Lin, et al., Adv. Funct. Mater., 2008, 18, 67-75.

39 Z. Ma, E. Wang, M. E. Jarvid, P. Henriksson, O. Inganas, F. Zhang and M. R. Andersson, J. Mater. Chem., 2012, 22, 2306-2314.
40 V. Bulovic, A. Shoustikov, M. A. Baldo, E. Bose, V. G. Kozlov, M. E. Thomoson and S. R. Forrest, Chem. Phys. Lett., 1998, 287, 455-460.

41 Z. R. Grabowski, K. Rotkiewicz and W. Rettig, Chem. Rev., 2003, 103, 3899-4032.

42 C. Liu, Q. Fu, Y. Zou, C. Yang, D. Ma and J. Qin, Chem. Mater., 2014, 26, 3074-3083.

43 E. Lippert, W. Lüder and H. Boos, in Advances in molecular spectroscopy, ed. A. Mangini, Pergamon Press, Oxford, 1962, p. 443.

44 (a) S. P. Jagtap, S. Mukhopadhyay, V. Coropceanu, G. L. Brizius, J. Bré das and D. M. Collard, J. Am. Chem. Soc., 2012, 134, 7176-7185; (b) S. Shirai, S. Iwata, T. Tani and S. Inagaki, J. Phys. Chem. A, 2011, 11(5), 7687-7699.

45 M. R. Zhu and C. L. Yang, Chem. Soc. Rev., 2013, 42, 4963-4976. 46 H. H. Chou, Y. H. Chen, H. P. Hsu, W. H. Chang, Y. H. Chen and C. H. Cheng, Adv. Mater., 2012, 24, 5867-5871.

47 Q. Zhang, J. Li, K. Shizu, S. Huang, S. Hirata, H. Miyazaki and C. Adachi, J. Am. Chem. Soc., 2012, 134, 14706-14709.

48 (a) M. Segal, M. Singh, K. Rivoir, S. Difley, T. V. Voorhis and M. A. Baldo, Nat. Mater., 2007, 6, 374-378; (b) W. Barford, Phys. Rev. B, 2004, 70, 205204-205208.

49 W. Jiang, L. Duan, J. Qiao, et al., Org. Lett., 2011, 13, 3146-3149. 50 Y. Liu, L. S. Cui, M. F. Xu, X. B. Shi, D. Y. Zhou, Z. K. Wang, Z. Q. Jiang and L. S. Liao, J. Mater. Chem. C, 2014, 2, 24882495.

51 Y. H. Lou, M. F. Xu, L. Zhang, Z. K. Wang, S. Naka, H. Okada and L. S. Liao, Org. Electron., 2013, 14, 2698-2704.

52 Z. Wang, Y. Lou, S. Naka and H. Okada, Appl. Phys. Lett., 2011, 98, 063302-063304.

53 V. Jankus, C. J. Chiang, F. Dias and A. P. Monkman, Adv. Mater., 2013, 25, 1455-1459.

54 J. Yang, Q. Guo, J. Wang, Z. Ren, J. Chen, Q. Peng, D. Ma and Z. Li, Adv. Optical Mater., 2018, 1800342.

55 H. Liu, Q. Bai, L. Yao, H. Zhang, H. Xu, S. Zhang, W. Li, Y. Gao, J. Li, P. Lu, H. Wang, B. Yang and Y. Mac, Chem. Sci., 2015, 6, 3797-3804.

56 (a) C. Wang, X. Li, Y. Pan, S. Zhang, L. Yao, Q. Bai, W. Li, P. Lu, B. Yang, S. Su and Y. Ma, ACS Appl. Mater. Interfaces, 2016, 8(5), 3041-3049; (b) B. Wang, X. Lv, J. Tan, Q. Zhang, Z. Huang, W. Yi and L. Wang, J. Mater. Chem. C, 2016, 4, 8473-8482; (c) Y. Zou, J. H. Zou, T. L. Ye, H. Li, C. L. Yang, H. B. Wu, D. G. Ma, J. G. Qin and Y. Cao, Adv. Funct. Mater., 2013, 23, 1781-1788.

57 (a) Z. Wang, P. Lu, S. Chen, Z. Gao, F. Shen, W. Zhang, Y. Xu, H. S. Kwok and Y. Ma, J. Mater. Chem., 2011, 21, 5451-5456; (b) H. Huang, Y. Wang, B. Wang, S. Zhuang, B. Pan, X. Yang, L. Wang and C. Yang, J. Mater. Chem. C, 2013, 1, 5899-5907; (c) Z. Wang, Y. Feng, H. Li, Z. Gao, X. Zhang, P. Lu, P. Chen, Y. Ma and S. Liu, Phys. Chem. Chem. Phys., 2014, 16, 1083710843; (d) Z. Gao, Z. Wang, T. Shan, Y. Liu, F. Shen, Y. Pan, H. Zhang, X. He, P. Lu, B. Yang and Y. Ma, Org. Electron., 2014, 15, 2667-2676. 